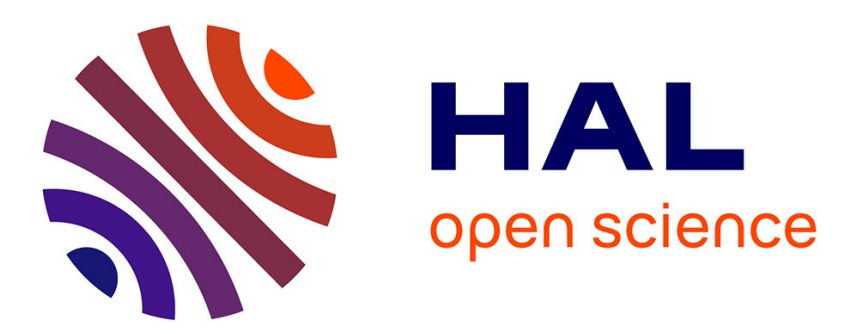

\title{
Studying divertor relevant plasmas in the Pilot-PSI linear plasma device: experiments versus modelling
}

K Ješko, Y. Marandet, H. Bufferand, J. Gunn, H van Der Meiden, G. Ciraolo

\section{To cite this version:}

K Ješko, Y. Marandet, H. Bufferand, J. Gunn, H van Der Meiden, et al.. Studying divertor relevant plasmas in the Pilot-PSI linear plasma device: experiments versus modelling. Plasma Physics and Controlled Fusion, 2018, 60 (12), pp.125009. 10.1088/1361-6587/aae80d . hal-02119026

\section{HAL Id: hal-02119026 \\ https://hal.science/hal-02119026}

Submitted on 10 May 2019

HAL is a multi-disciplinary open access archive for the deposit and dissemination of scientific research documents, whether they are published or not. The documents may come from teaching and research institutions in France or abroad, or from public or private research centers.
L'archive ouverte pluridisciplinaire HAL, est destinée au dépôt et à la diffusion de documents scientifiques de niveau recherche, publiés ou non, émanant des établissements d'enseignement et de recherche français ou étrangers, des laboratoires publics ou privés. 


\title{
Studying divertor relevant plasmas in the Pilot-PSI linear plasma device: Experiments vs. modelling
}

\author{
September 7, 2018
}

\author{
K. Ješko ${ }^{1,2}$, Y. Marandet ${ }^{3}$, H. Bufferand ${ }^{2}$, J. P. Gunn ${ }^{2}$, H. J. van der Meiden ${ }^{1}$, \\ G. Ciraolo ${ }^{2}$ \\ 1 DIFFER - Dutch Institute for Fundamental Energy Research, De Zaale 20, 5612 AJ \\ Eindhoven, the Netherlands \\ ${ }^{2}$ CEA, IRFM, F-13108 Saint-Paul-Lez-Durance, France \\ ${ }^{3}$ Aix-Marseille Univ., CNRS, PIIM, UMR 7345, F-13397 Marseille Cedex 20, France
}

\begin{abstract}
Predictions for the operation of tokamak divertors are reliant on edge plasma simulations typically consisting of a fluid plasma code in combination with a Monte Carlo code for neutral species. Pilot-PSI is a linear device operating with a cascaded arc plasma source that produces plasmas comparable to those expected during the inter-ELM phase in the ITER divertor $\left(T_{e} \sim 1 \mathrm{eV}, n_{e} \sim 10^{20} \mathrm{~m}^{-3}\right)$. In this study, plasma discharges in Pilot-PSI have been modelled using the Soledge2D fluid plasma code [1] coupled to the Eirene neutral Monte Carlo code [2] in order to a) investigate which phenomena need to be included in the modeling to reproduce experimental trends and b) provide new insights to the interpretation of experiments. The simulations highlight the key role of ion/molecule elastic collisions in determining the ion flux reaching the target. Recombination is likely to play a role at high molecular background pressure. However, even with the most advanced atomic and molecular model used in this work, $T_{e}$ at the target is overestimated with respect to the measurements using TS and spectroscopy. $T_{e}$ in the simulations appears to saturate at $0.7 \mathrm{eV}$ for a wide range of parameters, while experimentally values of $0.1-0.3$ $\mathrm{eV}$ are found. As a consequence, in the simulations the volume recombination is underestimated, which is a strong function of $T_{e}$ when it is below $1 \mathrm{eV}$. Further analysis of simulation results using a two-point formalism shows that inelastic collisions between electrons and neutral background particles remove most of the energy
\end{abstract}


flux, mainly via dissociation of molecules and molecular ions. However this happens mostly in the upstream region of the beam where $T_{e}>1 \mathrm{eV}$. For $T_{e}<1 \mathrm{eV}$, there seems to be no significant energy removal mechanism in the simulated cases. The results also indicate that conclusions on the importance of volume processes, e.g. recombination, cannot be solely based on $T_{e}$ or the dominance of certain reaction rate coefficients over others, but rather the complete transport picture, including macroscopic flow, has to be taken into account. In the cases studied here, the plasma is typically advected to the wall too fast for recombination to remove a significant fraction of the particle flux.

\section{Introduction}

The power transported from the core plasma through the separatrix is channelled to the plasma-facing componets (PFCs) via the scrape-off layer (SOL). The thickness of the SOL mapped to the outer mid-plane is of the order of several millimeters and for ITER it has been predicted to be $\sim 1 \mathrm{~mm}$ [3], leading to a total plasma wetted area of $\sim 2.3$ $\mathrm{m}^{2}$ [4] (this includes geometrical effects like flux expansion and inclination of divertor targets). During D-T operation, the power entering the SOL is expected to be $\sim 100$ MW, leading to an average heat flux density of $\sim 40 \mathrm{MW} / \mathrm{m}^{2}$ in case of no mitigation. On the other hand, the technological limit for steady-state power loading of ITER plasma facing components is $10 \mathrm{MW} / \mathrm{m}^{2}[4]$ ). This mismatch of expected and tolerable power fluxes is a major challenge on the way to harnessing fusion energy. The excess power has to be radiated away either by neutral hydrogenic or impurity species, ultimately leading to divertor plasma detachment [5-7], a regime in which both particle and power fluxes to divertor targets are strongly reduced. Understanding the key processes at play in divertor detachment is mandatory in order to optimize divertor performance.

\subsection{Linear devices as divertor simulators}

Linear plasma devices have been used extensively as divertor simulators in the past. A very important aspect of linear devices is that their cost per shot is significantly lower than in standard tokamaks, due to their inherently simpler construction and staff requirements. First experiments simulating and demonstrating the feasibility of a gaseous divertor concept were performed at the QED device $[8,9]$, showing strong reduction of power flux to a solid target in the last chamber of the device by increasing the neutral pressure in the chamber. This reduction was attributed to ions diffusing radially due to elastic collisions with neutral molecules, which were fed into the chamber. In similar experiments at PISCES [10], strong reduction of heat flux to the target was also found with increase of target chamber gas pressure, explained by anomalous radial transport. A significant body of knowledge originates from the Nagdis linear device. In [11-13], based on spectroscopy and comparison with the CRAMD collisional radiative model [14], 
molecular activated recombination is identified as an important process that reduces the particle flux arriving at the target, in $\mathrm{H} / \mathrm{He}$ mixture plasmas. However, the importance of radial transport is also pointed out in [15]. Additionally, the effect of transients on a detached plasma are assessed in [16]. Other devices with divertor physics research include TPD-I, MAP-2 and GAMMA10/PDX and progress on these is reviewed in [17].

In this work, we study a divertor-relevant plasma in the Pilot-PSI device. Pilot-PSI can provide densities of $10^{20}-10^{21} \mathrm{~m}^{-3}$, which is about one order of magnitude higher than typical plasmas produced by the other devices mentioned before, and more relevant to the regimes expected in ITER. The typical electron temperature $\left(T_{e}\right)$ close to the plasma source is $3-5 \mathrm{eV}$ in a hydrogen plasma.

\subsection{Relevance of linear devices to divertor physics}

The relevance of experiments in linear devices to tokamak divertors is often discussed, e.g. in [6]. Indeed, in a tokamak SOL, $T_{e}$ upstream, e.g. at the midplane separatrix, is $\sim 100$ $\mathrm{eV}$, while $T_{e}$ at the divertor target can be even below $1 \mathrm{eV}$ under detached conditions. Clearly, Pilot-PSI cannot access the high upstream temperatures. Therefore, Pilot-PSI can only mimic the area adjacent to the targets, where $T_{e}$ is low. More subtle differences that might not be so evident are related to the physics of particle balance: under high recycling and detached conditions in tokamaks, most of the particle source is concentrated in a "recycling region", poloidally located between X-point and the target plate. The ionization is maintained by power arriving via conduction from further upstream (which is a fraction of the auxiliary heating power). In Pilot-PSI the particle source is maintained by the cascaded arc, and a part of this plasma exhausts into the target chamber. The operation of the cascaded arc is de-coupled from what is happening in the target chamber, as will be seen later. Another difference is that the neutral background pressure $P_{n}$ in the vessel is set by the inflow of residual gas from the cascaded arc discharge chamber, since only about $10 \%$ of the gas fed into the discharge chamber is exhausted to the vessel in the form of plasma. In a tokamak, all neutrals in the divertor originate from plasma recombination, primarily on the solid surface. This is illustrated by the fact that the divertor neutral pressure is strongly coupled to the target particle flux, while in PilotPSI it is not the case. For the sake of completeness, it is important to note that for deeply detached divertors, a significant fraction of recombination can take place in the volume $[18,19]$.

Regarding the differences between divertors and linear plasma machines it is evident that a direct extrapolation of results from one to the other is difficult. However, it is still valuable to apply an edge transport suite to Pilot-PSI. Firstly, it can be checked how accurately the code reproduces trends observed experimentally (i.e. code-experiment benchmarking), possibly pointing at additional missing physics in the code. Secondly, the code can give good insights into which atomic/molecular (or other) processes are responsible for the reduction of power and particle fluxes to the target, i.e. do the book-keeping between 
the various mechanisms that drive flows in the plasma, i.e. ionisation/recombination, parallel and perpendicular transport etc.

Several linear devices have been modelled previously using different code packages. For instance, Pilot-PSI has been simulated using the B2.5-Eunomia code [20], Eunomia being a neutral Monte-Carlo (MC) code specifically developed for the geometry of linear devices. An advantage of the B2.5-Eunomia suite is a built-in collisional-radiative model that can calculate line emission using the local $T_{e}$ and also the population densities of vibrational states. Next, simulations for Magnum-PSI have been carried out by B2.5Eirene [21]. However, these simulations were performed before the Magnum-PSI device was launched and therefore without input from experiments. In the simulations, typically higher temperatures were obtained compared to the actual operational characteristics of Magnum-PSI [22]. Moreover, the geometry of the device assumed in the simulations does not correspond to the final layout. In another instance [23], the PSI-2 linear plasma device was simulated by the B2.5-Eirene package in a non-homogeneous magnetic field. In this work, it was found that inhomogeneities in the magnetic field can drive supersonic transitions in the plasma beam and also that the choice of artificial flux limiters used in the fluid code can significantly influence results in these low temperature cases. In a more recent study [24], a new transport code LINDA for linear devices is introduced and used to assess cooling efficiencies of various noble gases in the end cell of the GAMMA-10/PDX tandem mirror, identifying xenon as the most efficient radiator. As a general feature, using transport codes in linear geometry can help to shed light on features that could remain hidden or cannot be easily interpreted due to the inherently complex geometry of tokamaks. Linear devices offer a simple, yet still physical model system on which transport codes can be tested and ultimately also compared to experiments.

\section{Experimental setup}

\subsection{The Pilot-PSI linear device}

A schematic of the Pilot-PSI linear plasma device [25] is depicted in Fig. 1. It employs a high pressure cascaded arc discharge source [26] for plasma generation. A steady-state gas flow is fed into the discharge channel, with typical values between 1.5 - 3.0 standard liter per minute $(\mathrm{slm})$. The arc operation is sustained by a negative voltage on a hot cathode. The distance between the cathode and the grounded anode is $54 \mathrm{~mm}$ and the diameter of the discharge channel is $16 \mathrm{~mm}$ (in Figure 1, the cascaded arc source is not drawn to scale). Typical achievable discharge currents for steady state source operation are 100 200 A. The plasma then exhausts into the vessel and is transported to an actively cooled solid target, located approximately $0.56 \mathrm{~m}$ from the source nozzle. The plasma is confined by an axial magnetic field generated by a set of five coils. The magnetic field inside the vessel is homogeneous and can be varied in the range $0.2-1.6 \mathrm{~T}$. In the experiments shown in this work, only the $0.2 \mathrm{~T}$ setting was used. The low field reduces Ohmic heating effects 
which can cause post-heating of the plasma beam downstream from the source [27]. The pumping system is situated at the back of the vessel, the background neutral pressure in the vessel is set by the pumping rate and the inflow of the residual neutrals from the source and is typically of the order of several Pa. A cylindrical R- $\phi-\mathrm{Z}$ coordinate system is used to describe the system, where the Z-coordinate is aligned with the magnetic field and is the axis of symmetry of the plasma beam and $z=0$ is situated at the exit of the source discharge channel and the target is located at $\mathrm{z}=56 \mathrm{~cm}$.

\subsection{Diagnostics}

The key diagnostic was Thomson scattering which was performed at two axial locations (at $\mathrm{z}=4 \mathrm{~cm}$ and $\mathrm{z}=54 \mathrm{~cm}$, referred to as "upstream" and "target" locations, respectively) and is particularly suited to measure low temperature plasmas in the range $0.07 \mathrm{eV}$ to $35 \mathrm{eV}$ [28] with a radial spatial resolution of $0.6 \mathrm{~cm}$. The system uses an Nd:YAG laser operating at the second harmonic, $532 \mathrm{~nm}$. The scattered light detection is performed with an image intensifier and an ICCD camera. The system is capable of measuring electron density and temperature profiles of a plasma column of $30 \mathrm{~mm}$ in diameter with a spatial resolution of $0.6 \mathrm{~mm}$.

A single Langmuir probe was embedded in the target with a collecting area of circular shape and a diameter of $2 \mathrm{~mm}$. The probe area was perpendicular to the magnetic field lines, minimising effects of the magnetic pre-sheath which tend to increase the effective collection area of the probe, especially at low grazing angles of the magnetic field [29]. As will be seen later, the probe setup used here gave good agreement with the target Thomson scattering measurements, possibly also due to the normal incidence of the B-field.

Optical emission spectroscopy was performed at the same location as target Thomson scattering, i.e. at $\mathrm{z}=54 \mathrm{~cm}$, in the near UV spectral range 370 - $440 \mathrm{~nm}$, using one channel of an Avantes ULS2048 spectrometer. The line of sight was perpendicular to the beam (Fig. 1), focused on the central part.

The background neutral pressure, which we will denote as $P_{n}$, was measured by a capacitance manometer located at a port about $20 \mathrm{~cm}$ radially outwards from the axis of symmetry.

\section{Simulation setup}

\subsection{The Soledge2D fluid code}

The Soledge2D transport code was designed for investigation of the transport of multifluid plasmas in the tokamak edge and in the SOL [1]. The equations solved by the code with the setup used in this work are the following: 


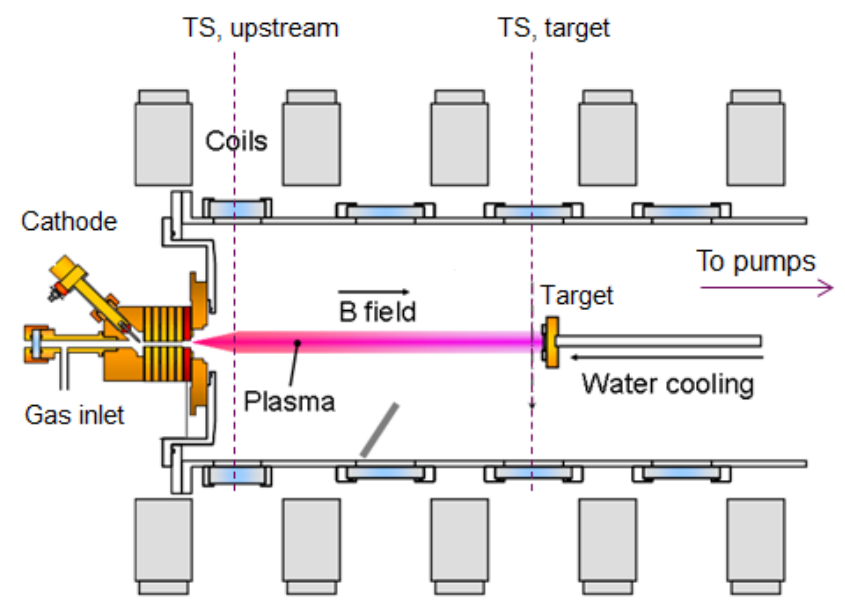

Figure 1: Schematic layout of the Pilot-PSI linear plasma device, with the two positions where radial profile measurements using Thomson scattering can be performed.

$$
\begin{gathered}
\frac{\partial n}{\partial t}+\vec{\nabla} \cdot\left(n u_{\|} \vec{b}\right)=\vec{\nabla} \cdot\left(D \vec{\nabla}_{\perp} n\right)+S_{n}^{(N)}+S_{n}^{(\mathrm{ext})} \\
\frac{\partial n u_{\|}}{\partial t}+\vec{\nabla} \cdot\left(n u_{\|}^{2} \vec{b}\right)+\nabla_{\|}\left(n k_{B} \frac{T_{e}+T_{i}}{m_{i}}\right)=\vec{\nabla} \cdot\left(\nu \vec{\nabla}_{\perp} n u_{\|}\right)+S_{G}^{(N)} \\
\frac{\partial}{\partial t}\left(\frac{3}{2} k_{B} n T_{i}+\frac{1}{2} m_{i} u_{\|}^{2}\right)+\vec{\nabla} \cdot\left(\frac{5}{2} k_{B} n u_{\|} T_{i} \vec{b}+\frac{1}{2} m_{i} n u_{\|}^{3} \vec{b}+\kappa_{i}^{0} T_{i}^{5 / 2} \nabla_{\|} T_{i} \vec{b}\right)+u_{\|} \nabla_{\|}\left(n k_{B} T_{e}\right)= \\
\vec{\nabla} \cdot\left(\left(\frac{3}{2} k_{B} T_{i}+\frac{1}{2} m_{i} u_{\|}^{2}\right) D \overrightarrow{\nabla_{\perp}} n+\frac{3}{2} k_{B} \chi_{\alpha} n \overrightarrow{\nabla_{\perp}} T_{i}+\nu n \vec{\nabla}_{\perp}\left(\frac{1}{2} m_{i} u_{\|}^{2}\right)\right)+Q_{e i}^{(c)}+S_{E, i}^{(N)}+S_{E, i}^{(\mathrm{ext})} \\
\frac{\partial}{\partial t}\left(\frac{3}{2} k_{B} n T_{e}\right)+\vec{\nabla} \cdot\left(\frac{5}{2} k_{B} n u_{\|} T_{e} \vec{b}+\kappa_{e}^{0} T_{e}^{5 / 2} \nabla_{\|} T_{e} \vec{b}\right)-u_{\|} \nabla_{\|}\left(n k_{B} T_{e}\right)= \\
+\frac{3}{2} k_{B} \vec{\nabla} \cdot\left(T_{e} D \vec{\nabla}_{\perp} n+\chi_{\alpha} n \vec{\nabla}_{\perp} T_{e}\right)-Q_{e i}^{(c)}+S_{E, e}^{(N)}+S_{E, e}^{(\mathrm{ext})}
\end{gathered}
$$

where $n$ is the plasma density $\left(n=n_{e}=n_{i}\right), T_{i}$ and $T_{e}$ are the ion and electron tempertures, respectively, $\vec{b}$ is the unit vector in the direction of the magnetic field and the operators are defined in the following way: $\nabla_{\|}=\vec{b} \cdot \vec{\nabla}$ and $\vec{\nabla}_{\perp}=\vec{\nabla}-\vec{b} \nabla_{\|}$. The term $Q_{e i}^{(c)}$ represents the coupling between electrons and ions and has the form $Q_{e i}^{(c)}=$ $\beta n^{2} T_{e}^{-3 / 2}\left(T_{i}-T_{e}\right)$, where $\beta=\frac{\sqrt{m}_{e}}{m_{i}} 4 \sqrt{\pi} e^{4} Z^{2} \ln \Lambda$. The anomalous perpendicular transport coefficients $D, \nu$ and $\chi_{i}, \chi_{e}$ are for density, parallel momentum and ion/electron energy, 


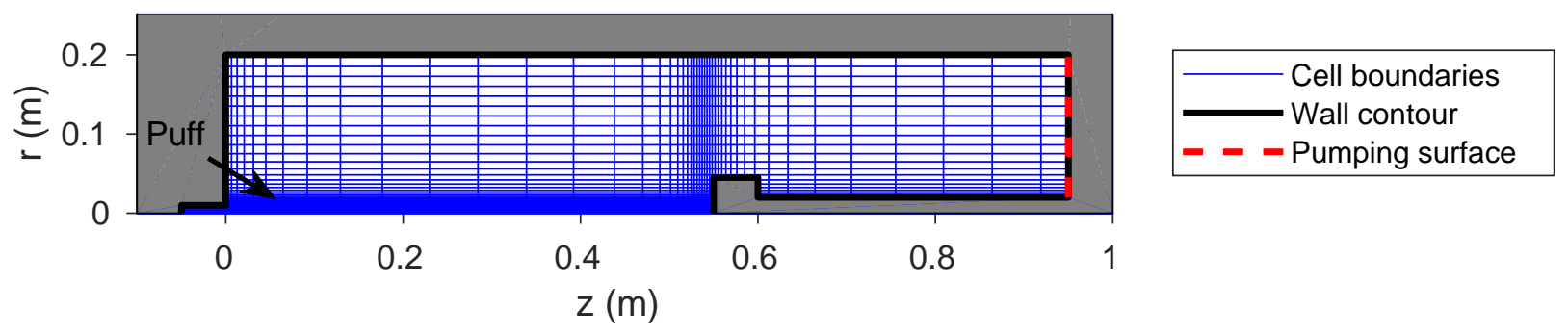

Figure 2: The Pilot-PSI non-uniform grid used in the Soledge2D-Eirene simulations.. The small region demarcated by $0 \mathrm{~cm}<R<1 \mathrm{~cm}$ and $-5 \mathrm{~cm}<Z<0 \mathrm{~cm}$ is a "source region", where the external volumetric source terms based on formula 5 are prescribed. The pumping surface is located at the back of the vessel, where an absorption probability (albedo) for impinging particles can be defined. The recycling coefficient in the "source region" is set to 0 for reasons described in the text. The remainder of the vessel walls use a recycling coefficient $R=1$. The gas puff is located at $(R, Z)=(1.005,0.000) \mathrm{cm}$.

respectively, and are uniform in the whole simulation domain throughout this work. The terms $S_{n}^{(N)}, S_{G}^{(N)}$ and $S_{E, \alpha}^{(N)}$ are sources of particles, parallel momentum and energy due to neutral particles, respectively. These source terms due to neutrals are calculated by the kinetic Monte-Carlo (MC) code Eirene [2], which will be described in section 3.2. The terms $S_{n}^{(e x t)}, S_{G}^{(e x t)}, S_{E, i}^{(e x t)}, S_{E, e}^{(e x t)}$ represent externally forced sources of plasma particles, momentum and energy, and are used to generate the plasma beam in this setup of the code. Their exact form is discussed in the end of this section. Drifts, electric fields and currents are implemented in Soledge2D [30] but were turned off in all simulations shown in this work since the focus was on atomic and molecular physics, i.e. the interaction of the plasma beam and the neutral background.

The geometry of the linear plasma device may seem very different from a tokamak, however they have common aspects, which enable to create a field-aligned grid that the code can directly use. The grid for the linear device can be regarded as a subspace of a tokamak grid. It is in fact topologically equivalent to a scrape-off layer with no toroidal field, i.e. the plasma is simulated up to the axis of symmetry. The axial magnetic field of the linear device corresponds to the poloidal magnetic field of a tokamak and has a constant value of $0.2 \mathrm{~T}$ in all simulations presented here, in line with the experiment. The grid used in the simulations is depicted in Fig. 2. Additionally, a variable grid density is used in order to provide high resolution in the plasma beam and close to the walls, while in areas of less interest the cells are larger, to save computational time.

The plasma wall interaction is treated using the penalization technique [31-34], a unique feature of SolEdge2D permitting simulation of the plasma up to the first wall in tokamaks. Standard Bohm boundary conditions and sheath heat transmission are imposed at the plasma-wall interface, i.e. $|M| \geq 1$ and $q_{t, e}=\gamma_{e} n M c_{s} T_{e}$, where $M$ is the 
Table 1: Values of various constants used for the external source terms (formula 5) for the reference case

\begin{tabular}{llllll} 
Type & $S_{i}^{(\text {ext,tot })}$ & $\lambda_{r}(\mathrm{~mm})$ & $\lambda_{z}(\mathrm{~mm})$ & $r_{0}(\mathrm{~mm})$ & $z_{0}(\mathrm{~mm})$ \\
\hline$S_{n}^{(\text {ext })}$ & $4.1 \times 10^{20} \mathrm{~m}^{-3} \mathrm{~s}^{-1}$ & 5.0 & 10.0 & 0 & -25 \\
$S_{E e}^{(\text {ext })}$ & $1.2 \times 10^{3} \mathrm{Wm}^{-3}$ & 3.5 & 7.1 & 0 & -25 \\
$S_{E i}^{(\text {ext })}$ & $0.3 \times 10^{3} \mathrm{Wm}^{-3}$ & 3.5 & 7.1 & 0 & -25 \\
\hline
\end{tabular}

Mach number, $q_{t, e}$ is the energy flux density through the interface for electrons. For ions, the energy flux density is given by $q_{t, i}=n M c_{s}\left(\gamma_{i} T_{i}+1 / 2 M^{2} c_{s}^{2}\right)$ since the energy of the mean flow has to be accounted for, where $c_{s}$ is the sound speed and $\gamma_{\alpha}$ are the sheath heat transmission factors for ions and electrons, set to 2.5 and 4.5, respectively. Note that even though Soledge2D energy equations are written in terms of the total energy, the values of heat transmission factors reported here are for the internal energy part. We assume the ion velocity distribution function at the sheath edge to be a shifted Maxwellian with $c_{s}$ normal to the wall, so the effective sheath heat transmission factor for ions is in fact 3.5 for $T_{i}=T_{e}$ (that is, on the high side). However, this choice ensures internal consistency of the code and is related to the coupling of Soledge2D and Eirene. On the axis of symmetry the boundary condition of vanishing perpendicular gradients is used. It should be noted that the PIC database connecting the magnetic pre-sheath entrance conditions to the ion velocity distribution at the wall available in Soledgde2D-Eirene [1] is not used here since the former is designed for grazing magnetic field incidence.

Anomalous values are assigned to the radial transport coefficients and a sensitivity study to the choice of these transport coefficients is performed in section 4.1.

It is beyond the scope of this contribution to model the details of the cascaded arc discharge self-consistently. This would require inclusion of the electric currents and drifts in the simulations, as well as thermionic emission from the hot cathode. However, the principal focus here lies in the interaction of the plasma beam with the surrounding neutral gas. Therefore, the plasma particle and power sources are directly prescribed as external volumetric source terms $S_{n}^{(e x t)}, S_{G}^{(e x t)}, S_{E, i}^{(e x t)}, S_{E, e}^{(e x t)}$ in the Soledge2D equations (1)-(3), section 3.1. The shape and magnitude of these is defined to match Thomson scattering profiles measured close to the source (shown in section 4.1). For example, the external volumetric source terms for the plasma ion/electron source has the form of a Gaussian function in both $r$ and $z$ directions:

$$
S_{n}^{(e x t)}(r, z)=\frac{S_{n}^{(e x t, t o t)}}{C} \exp \left(-\left(r-r_{0}\right)^{2} / \lambda_{r}^{2}\right) \exp \left(-\left(z-z_{0}\right)^{2} / \lambda_{z}^{2}\right),
$$

where $r_{s r c}, z_{s r c}$ are the positions of the profile maxima, $\lambda_{r}, \lambda_{z}$ are the profile widths, $C$ is a normalization constant such that the volume integral over the simulation domain be equal to the total number of injected particles, i.e. $\int_{V} S_{n}^{(e x t)} \mathrm{d} V=S_{n}^{(e x t, t o t)}$. A similar 
Table 2: List of atomic and molecular physics processes used in Eirene.

\begin{tabular}{cll} 
\# & Reaction & Event type \\
\hline$(1)$ & $\mathrm{H}+\mathrm{e} \rightarrow \mathrm{H}^{+}+2 \mathrm{e}$ & Electron impact ionization \\
$(2)$ & $\mathrm{H}+\mathrm{H}^{+} \rightarrow \mathrm{H}^{+}+\mathrm{H}$ & Charge exchange \\
$(3)$ & $\mathrm{H}_{2}+\mathrm{e} \rightarrow \mathrm{H}_{2}^{+}+2 \mathrm{e}$ & Electron impact ionization \\
$(4)$ & $\mathrm{H}_{2}+\mathrm{e} \rightarrow 2 \mathrm{H}+\mathrm{e}$ & Dissociation \\
$(5)$ & $\mathrm{H}_{2}+\mathrm{e} \rightarrow \mathrm{H}+\mathrm{H}^{+}+2 \mathrm{e}$ & Dissociative ionization \\
$(6)$ & $\mathrm{H}_{2}+\mathrm{H}^{+} \rightarrow \mathrm{H}_{2}+\mathrm{H}^{+}$ & Elastic collision \\
$(7)$ & $\mathrm{H}_{2}+\mathrm{H}^{+} \rightarrow \mathrm{H}_{2}^{+}+\mathrm{H}$ & Ion conversion \\
$(8)$ & $\mathrm{H}_{2}^{+}+\mathrm{e} \rightarrow \mathrm{H}^{+}+\mathrm{H}^{+}+2 \mathrm{e}$ & Dissociative ionization \\
$(9)$ & $\mathrm{H}_{2}^{+}+\mathrm{e} \rightarrow \mathrm{H}^{+}+\mathrm{H}+\mathrm{e}$ & Dissociation \\
$(10)$ & $\mathrm{H}_{2}^{+}+\mathrm{e} \rightarrow 2 \mathrm{H}$ & Dissociative recombination \\
$(11)$ & $\mathrm{H}^{+}+\mathrm{e} \rightarrow \mathrm{H}$ & Electron-ion recombination \\
\hline
\end{tabular}

external source term is also defined for the energy source on electrons and ions, $S_{E e}^{(e x t)}$ and $S_{E i}^{(e x t)}$, respectively. The position and spatial extent of the external volumetric source terms was chosen such that it stays well within the small area of the cascaded arc source. The values of the individual constants for the reference case are listed in Tab. 1. These are kept the same for all the other cases in this work.

\subsection{Eirene for neutral particles}

The interaction of plasma and neutrals is treated by the well-established Eirene MonteCarlo code [2]. In Pilot-PSI, neutrals enter the system by three channels 1) the residual gas entering the vessel from the cascaded arc source 2) main ion recycling, e.g. at the target and 3) recombination in the volume. The latter two are calculated self-consistently by Eirene, while the constant gas inflow rate is simulated as a constant puff of $\mathrm{H}_{2}$ at ambient temperature $(0.03 \mathrm{eV})$ at the location depicted in Fig. 2. In the experiment, this is an externally controllable quantity, and the value of the total source inflow was 2.5 standard liters per minute $(\mathrm{slm})$ corresponding to about $10^{21} \mathrm{H}_{2} / \mathrm{s}$ in all experiments presented here. This value is also used in the simulations. The recycling coefficient at the plasmawall interface is set to unity throughout all the simulations presented here, except for two locations: The pumping surface is located at the back end of the vessel, Fig. 2, where one can specify an absorption probability (albedo) for neutral particles. The absorption probability is set to match measurements of the neutral pressure in the vacuum vessel, typically in the range of several $\mathrm{Pa}$. The second region where the recycling coefficient is not unity is the source region, where it is set to 0 . Since the cascaded arc source is not modelled self-consistently, but rather by adding external source terms described in section 3.1 , formula 5 , it is of little interest to take into account recycling of ions on the walls of the source region. This would also render tweaking of the source terms for matching 

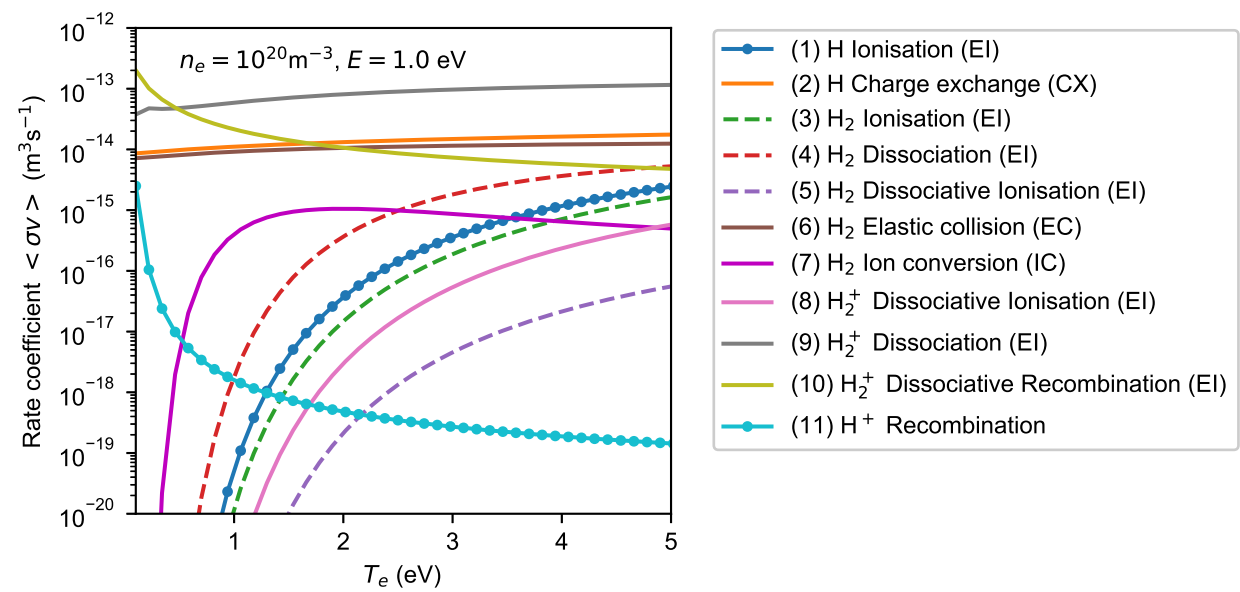

Figure 3: Plots of rate coefficients for different processes used in the atomic physics model in Eirene as a function of $T_{e}$ and for $n_{e}=10^{20} \mathrm{~m}^{-3}$ in case of density dependent rate coefficients (processes (1), (3-5) and (8-11)) and for a relative energy of $E=1 \mathrm{eV}$ for reactions (2) and (6)). The reaction numbering in the legend of the figure is the same as in Tab. 2. The rate coefficients are taken from the AMJUEL database (available from www.eirene.de) .

with upstream Thomson scattering profiles more challenging. The species considered in Eirene are hydrogen atoms $\mathrm{H}$ and molecules $\mathrm{H}_{2}$ and $\mathrm{H}_{2}^{+}$molecular ions. The latter is treated in the static approximation, i.e. its motion is not followed and the next collision is supposed to happen at the location of birth - in other words, $\mathrm{H}_{2}^{+}$is treated as a shortlived species. This assumption will be checked a posteriori in section 4.2. The atomic physics model used in Eirene is depicted in Tab. 2. This model is the same as the model described in [35], which was used extensively in predictive simulations for ITER [36], however, in our case neutral-neutral collisions and radiation opacity were not included. Including molecular processes is critical for reproduction of basic experimental features in Pilot-PSI. A plot of selected rate coefficients for reactions in Tab. 2 is shown in Fig. 3 in order to show which processes become dominant in which temperature regions for a given density. We point out here the importance of the ion conversion process (7) that is the dominant contributor to the formation of $\mathrm{H}_{2}^{+}$molecular ions in the $T_{e}$ range of interest. The molecular ion can then either dissociate into a main ion and an atom, reaction (9), or dissociatively recombine into two atoms. The latter process, including the preceding ion conversion, is termed molecular assisted recombination (MAR). The third possible reaction including $\mathrm{H}_{2}^{+}$, dissociative ionisation (8) is comparatively negligible in the $T_{e}$ range of interest. 


\section{Results \& Discussion}

\subsection{Understanding basic features}
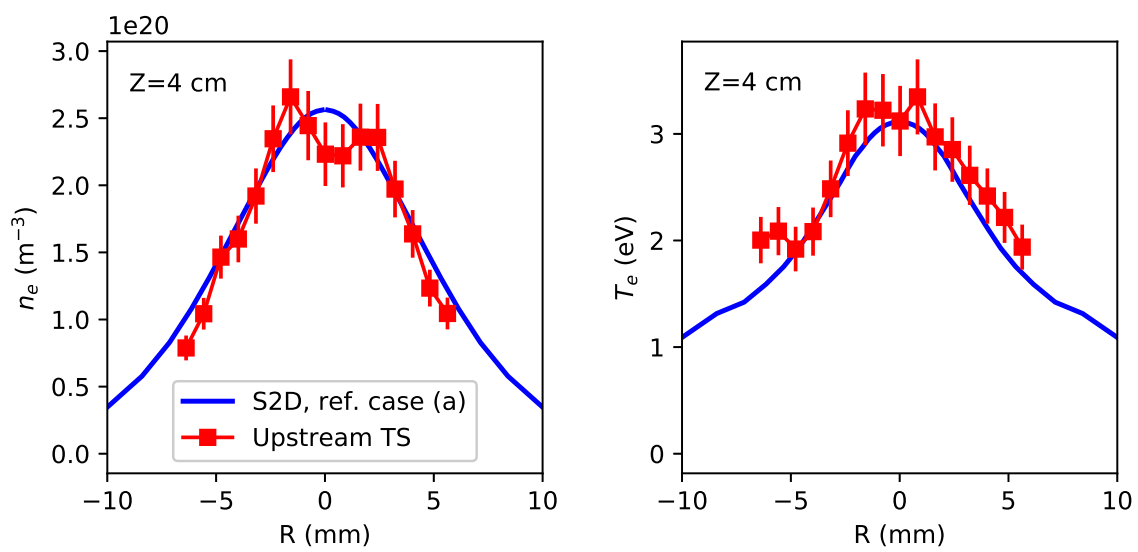

Figure 4: Comparison of upstream $n_{e}, T_{e}$ profiles measured by Thomson scattering and simulation results for the reference case (a). The match was obtained by adjusting the free parameters of the external volumetric source terms, see section 1.

Table 3: Radial transport coefficients and pumping albedos used in the simulations presented.

\begin{tabular}{clclcl} 
Case & $\begin{array}{l}D \\
\left(\mathrm{~m}^{2} / \mathrm{s}\right)\end{array}$ & $\begin{array}{c}\chi_{e}, \chi_{i} \\
\left(\mathrm{~m}^{2} / \mathrm{s}\right)\end{array}$ & $\begin{array}{l}\text { albedo } \\
\left(\times 10^{-2}\right)\end{array}$ & $\begin{array}{c}P_{n} \\
(\mathrm{~Pa})\end{array}$ & Remark \\
\hline (a) & 0.3 & 0.3 & 0.54 & 3.3 & ref. case \\
(b) & 0.3 & 1.0 & 0.54 & 3.4 & \\
(c) & 1.0 & 1.0 & 0.54 & 3.6 & \\
(d) & 0.1 & 0.1 & 0.54 & 3.2 & \\
(e) & 0.3 & 0.3 & 2.00 & 1.3 & \\
(f) & 0.3 & 0.3 & 1.20 & 1.9 & \\
(g) & 0.3 & 0.3 & 0.80 & 2.6 & \\
(h) & 0.3 & 0.3 & 0.40 & 4.1 & \\
(i) & 0.3 & 0.3 & 0.25 & 5.0 & \\
(j) & 0.3 & 0.3 & 0.17 & 6.0 & \\
(k) & 0.3 & 0.3 & 0.54 & 4.0 & el. coll. off
\end{tabular}

The reference case is defined by the input parameters in Tab. 3 and case (a). The sensitivity of the results to the choice of the transport coefficients is discussed in the next 

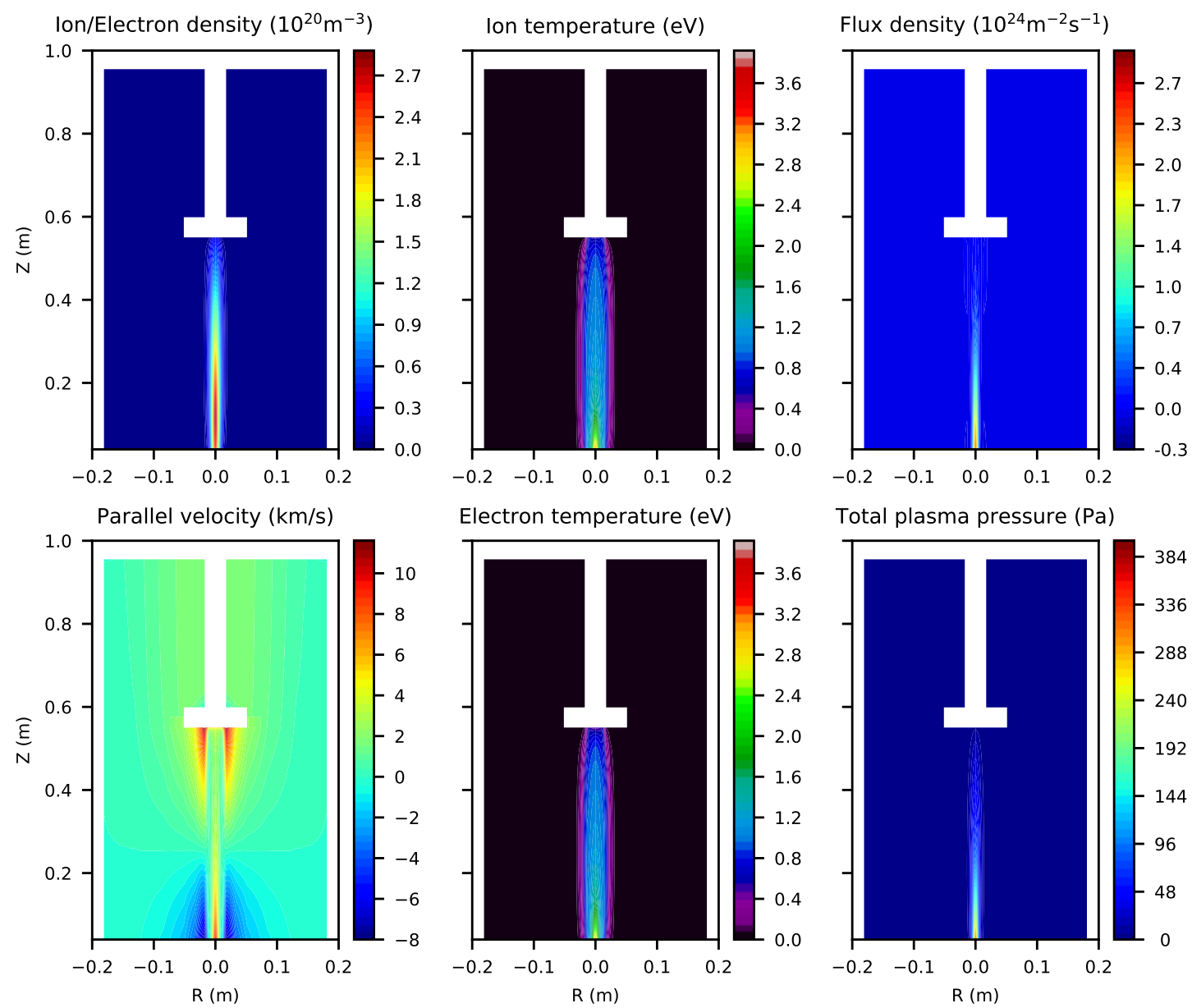

Figure 5: Plots of plasma parameters for the reference case (a). The blank areas correspond to space taken up by the target and vessel walls.

section (cases (b) to (e)). The pumping albedo was adjusted to match a background pressure of $P_{n}=3.2 \mathrm{~Pa}$. The external source terms were adjusted manually for the reference case until satisfactory agreement with measured upstream Thomson scattering profiles was achieved. The resulting upstream $(\mathrm{Z}=4 \mathrm{~cm})$ radial profiles of $n_{e}$ and $T_{e}$ of the reference case simulation are shown in Fig. 4 together with the upstream Thomson scattering measurement which were used for the matching. For all the other cases, the same parameters for the source terms were used. Next, 2-D maps of plasma parameters of the reference case (a) are shown on Fig. 5. As a general feature, there is a strong, monotonic axial reduction of all the quantities from upstream towards the target (with the exception of the parallel velocity, which is not monotonic, but decelerates in the usptream region of the beam and then accelerating towards the target again, which is expected from the 

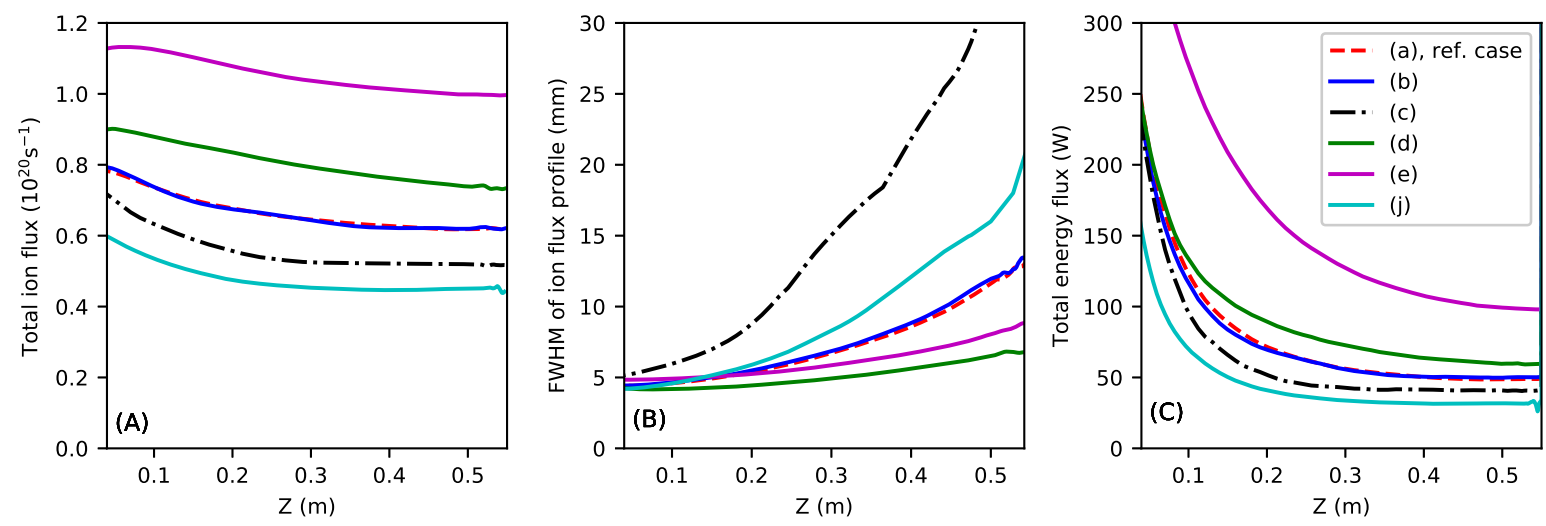

Figure 6: (A) Parallel profiles of the total, section integrated flux density, (B) parallel profile of the beam width (Full width half maximum of the radial flux density $\Gamma(r)$ ) and (C) parallel profile of the total, section integrated energy flux, listed for a number of different cases from Tab. 3 .

imposed boundary conditions). The total plasma pressure is the sum of the static and dynamic components, $P_{\text {tot }}=P_{\text {stat }}+P_{\text {dyn }}=n_{i} k_{B} T_{i}+n_{e} k_{B} T_{e}+n_{i} c_{s}^{2} M^{2}$. From the plot of the flux density $\Gamma$ and total plasma pressure $P_{\text {tot }}$, it may misleadingly seem that the beam is not reaching the target, however, this is just a consequence of the specific geometry and the imposed radial transport. In fact, in the reference case, most of the flux from the source plasma is reaching the target. This is shown in Fig. 6 (A), where the axial profile of the total, cross-section integrated flux density, $\Gamma_{\text {tot }}=\int_{0}^{2 \pi} \int_{0}^{R_{\text {vess }}} r \Gamma(r) \mathrm{d} r \mathrm{~d} \phi$ is plotted ( $R_{\text {vess }}$ is the radius of the vessel). $\Gamma_{\text {tot }}$ decreases only by about $20 \%$, meaning that there are net sinks of plasma between upstream and target locations (It was shown in [37] that MAR is the most efficient recombination channel for these cases.), however most of the flux still reaches the target. Instead, the strong reduction in the parallel ion flux density observed in Fig. 5 is driven mainly by the radial transport, which is effectively causing broadening of the beam, from about $\sim 4 \mathrm{~mm}$ upstream to $\sim 12 \mathrm{~mm}$ at the target, as can be seen in Fig. 6.

The sensitivity of the results to the choice of radial transport coefficients was also examined. Four different sets of transport coefficients were tested including the reference case and are listed in Tab. 3, cases (a)-(d). All other parameters, including the external volumetric source terms, were kept constant for these cases. From Fig. 6 (B), it can be seen that in case (b), where only the energy transport coefficients were changed, there is no significant difference neither in the axial profiles of the total ion flux, beam width and total energy flux. However, when the particle diffusion coefficient $D$ is changed, the beam width increases much faster for case (c), when $D=1 \mathrm{~m} / \mathrm{s}^{2}$ and slower when $D=0.1$ $\mathrm{m} / \mathrm{s}^{2}$. In terms of changes of total ion flux, Fig. 6 (A) it decreases roughly by similar amounts, between about $15-25 \%$. However, it is to be noted that the total initial ion 

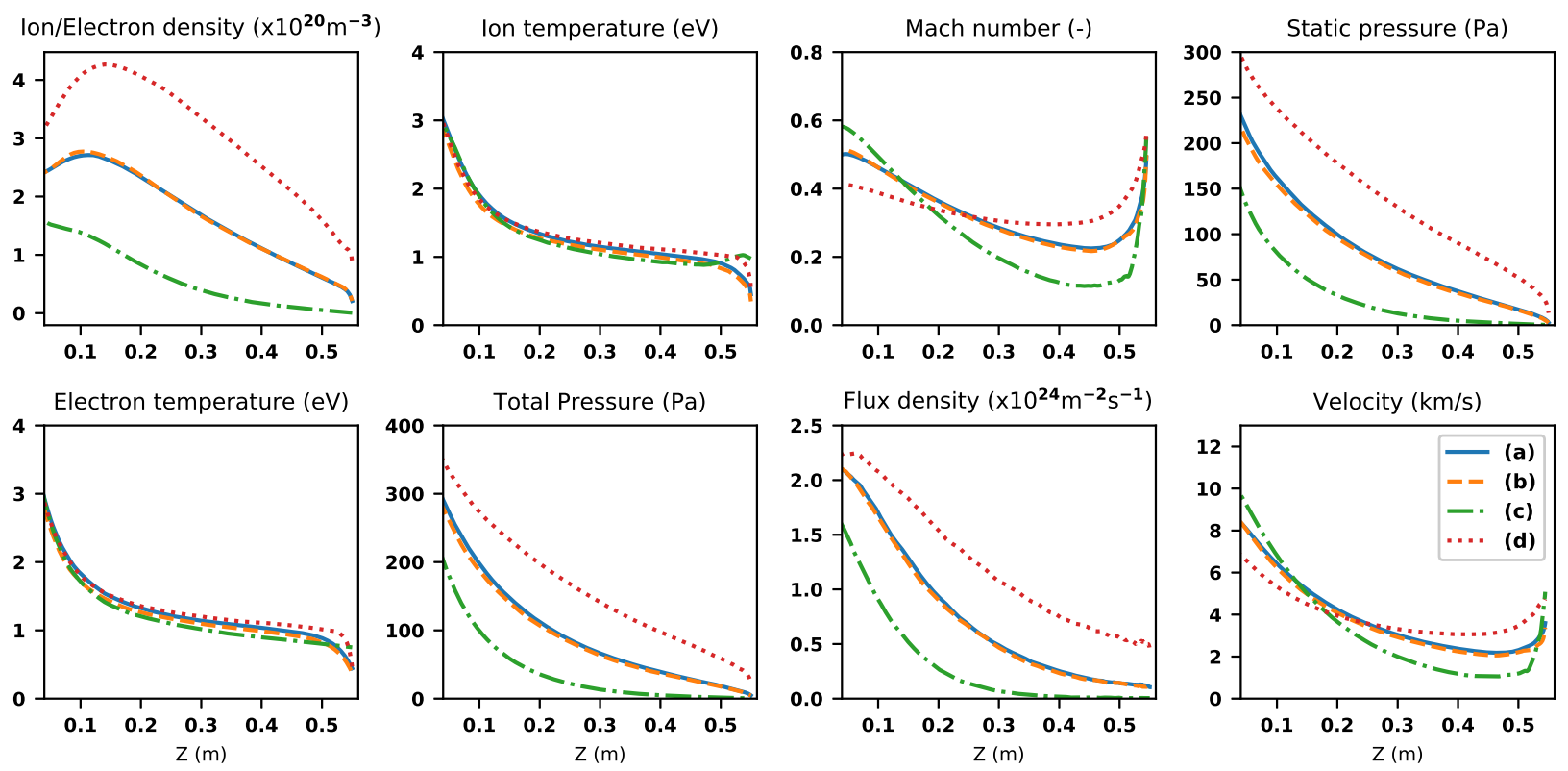

Figure 7: Parallel profiles of various plasma parameters on the flux tube located $1.4 \mathrm{~mm}$ from the axis of symmetry (Description of cases in Tab. 3).

fluxes (i.e. at the upstream location, $Z=4 \mathrm{~cm}$ ) are not identical. From Fig. 6 (C) it can be seen that most energy is reaching the target in the case $(d)$ (excluding case $(j)$ which belongs to a different scan), when the transport coefficients have the lowest values, and is about $60 \mathrm{~W}$. However, the difference between the other cases is not substantial, the amount being $50 \mathrm{~W}, 49 \mathrm{~W}, 41 \mathrm{~W}$ for cases (a), (b), (c), respectively.

Parallel profiles for the flux tube at $\mathrm{r}=1.4 \mathrm{~mm}$ are plotted in Fig. 7. The parallel profiles are more suitable for the interpretation of results compared to the 2D maps in figures 5 and 9 since they reveal also more subtle features. For the reference case (a), we have first a small increase of density up to $Z \simeq 0.1 \mathrm{~m}$, and after that a steady reduction towards the target plate. The increase of the density in this part is driven by a reduction in the parallel flow velocity, rather than ionisation sources in the volume. Although there is a positive source of plasma in the region (Fig. 8), the flux density profile, Fig. 7, is decreasing in this region (and also throughout the whole axial profile), meaning that the $\vec{\nabla} \cdot\left(D \vec{\nabla}_{\perp} n\right)$ term on the R.H.S. of the continuity equation 1 is stronger in absolute magnitude than the contribution of neutrals to the particle source $S_{n}^{(\mathrm{N})}\left(S_{n}^{(\text {ext })}=0\right.$ by definition here, Tab. 1). The $\vec{\nabla} \cdot\left(D \vec{\nabla}_{\perp} n\right)$ term can be looked at as a sink term arising from the presence of perpendicular transport. Since there is an axial drop in the flux density, the observed density increase must be compensated by a reduction in the parallel flow velocity, which is indeed the case. In the remaining part of the profile $0.1 \mathrm{~m}<$ $\mathrm{Z}<0.55 \mathrm{~m}$, we can see that the axial gradient in the parallel flow velocity is not so 

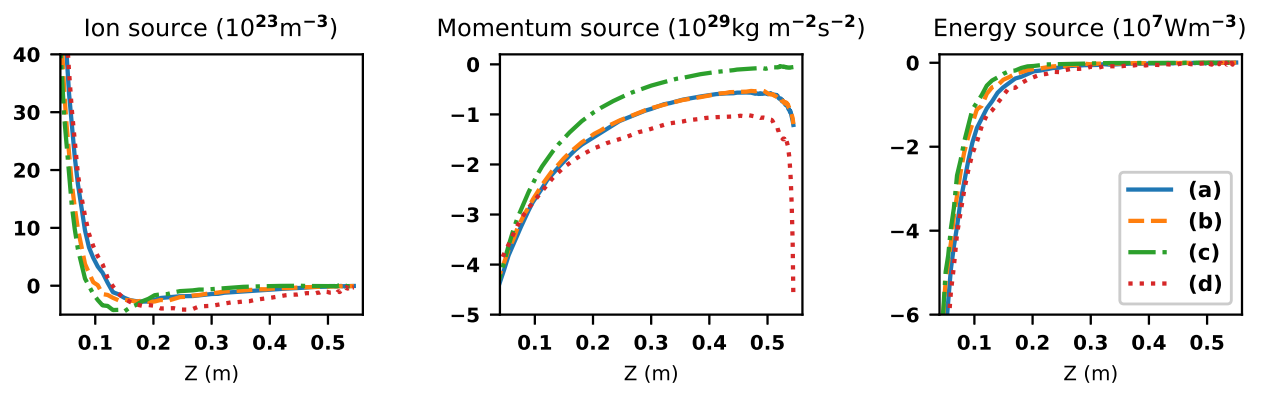

Figure 8: Parallel profiles of volumetric source terms of particles, momentum, and energy (sum of electron and ion energy source terms) due to neutral particles (Description of cases in Tab. 3).

pronounced, and it is too weak to compete with the sinks due to radial transport and interactions with neutrals, and as a consequence, also the density decreases in this part. In fact, in this region, the plasma is recombining, $S_{n}^{(\mathrm{N})}<0$, which is expected given that $T_{e}<2 \mathrm{eV}$ and $T_{e}$ dependencies of rate coefficients from Fig. 3.

In case (b), with the perpendicular energy diffusivities $\chi_{e}=\chi_{i}$ increased to $1 \mathrm{~m} / \mathrm{s}^{2}$, there is no significant change in the obtained profiles, indicating that the simulations are not sensitive to this parameter. However, in case (c) also the particle diffusion coefficient $D$ was increased to the same value, $1 \mathrm{~m} / \mathrm{s}^{2}$. This has a very strong influence on the observed profiles, mainly reducing the density, flux density and total plasma pressure. Indeed, the ion flux density at the target is now only a small fraction of the original flux density upstream. However, it is important to point out that the $T_{e}$ nor $T_{i}$ do not change much by increasing $D$.

Conversely, in case (d), all perpendicular diffusion coefficients have been reduced to 0.1 $\mathrm{m} / \mathrm{s}^{2}$. As expected, with the reduced radial transport, the plasma density and flux density are much higher than in the reference case (a). Again, the $T_{e}$ and $T_{i}$ profiles do not show a significant response to the change of the perpendicular transport coefficients, although one can see that in general the temperature is slightly higher than in the reference case (a). It is to be noted that here the perpendicular transport is strong enough to suppress the slight increase in density described in the previous paragraph for cases (a) and (b).

\subsection{Neutral inventory}

2D maps of various moments of species treated by Eirene are depicted in Fig. 9 for the reference case (a). It can be seen that the molecular density profile is hollow, with molecules depleted in the center of the beam. The remaining molecules in the beam are heated by the plasma to typical temperatures up to $1 \mathrm{eV}$. Hollow molecular profiles and heating of molecules was observed in Pilot-PSI in [38] and this is in line with the 

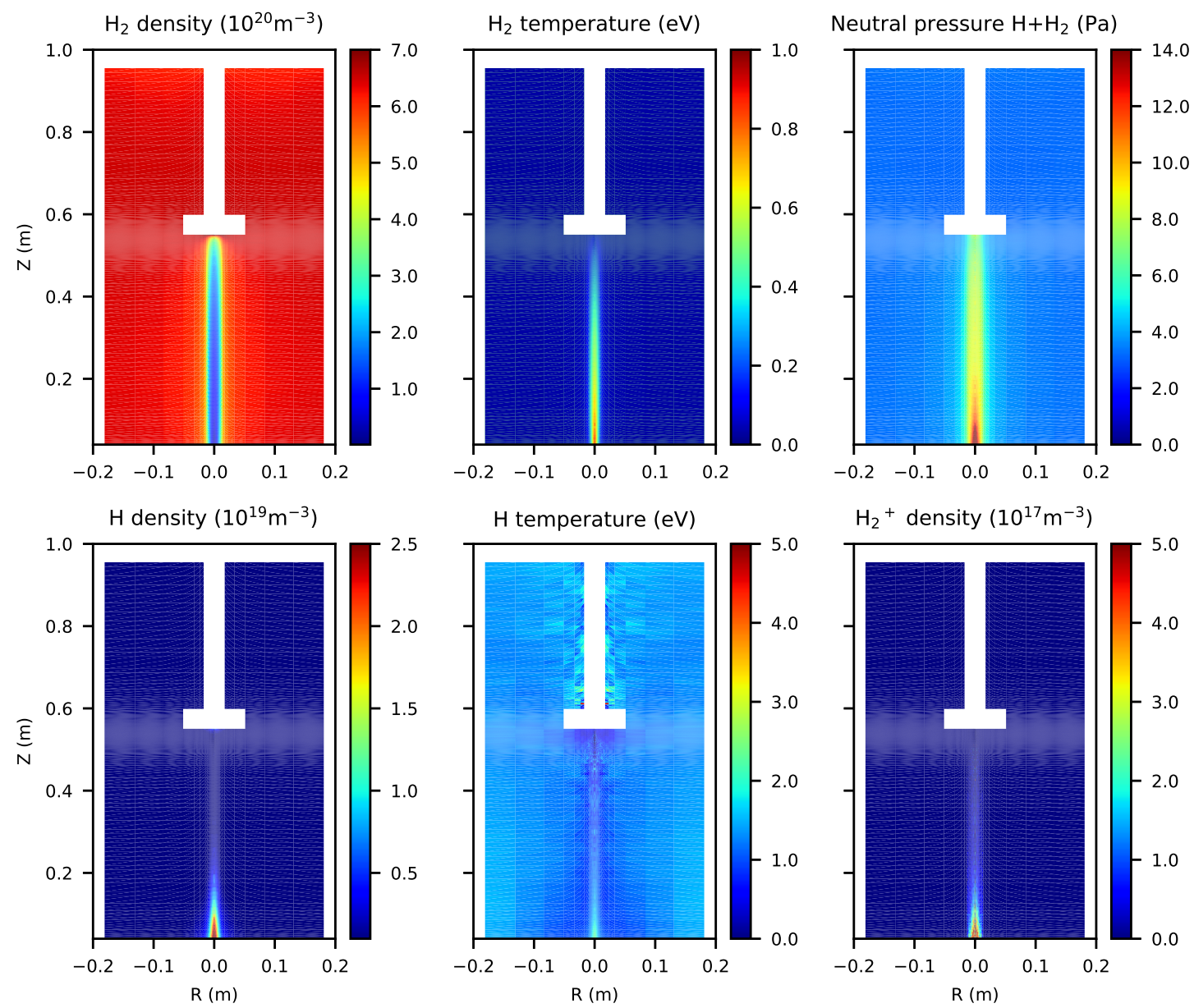

Figure 9: Plots of parameters of species treated by Eirene for the reference case (a). The blank areas correspond to space taken up by the target and vessel walls. The neutral pressure is the combined static pressure of atoms and molecules, $P_{n}=n_{\mathrm{H}} k T_{\mathrm{H}}+n_{\mathrm{H}_{2}} k T_{\mathrm{H}_{2}}$. The trajectories of molecular ions are not followed in Eirene, they are instantaneously followed by reactions (8), (9) or (10), Tab. 2.

modelling. On the other hand, the $\mathrm{H}$ atoms are mainly present in the upstream area of the plasma, where the plasma is hot enough for dissociation of molecules and molecular ions to be efficient. The density of molecular ions is significantly lower than the typical plasma, molecule and atomic densities, and peak values are of the order $10^{17} \mathrm{~m}^{-3}$, concentrated in upstream areas of the beam. The neutral static pressure $P_{n}$ is uniform outside of the plasma beam and is elevated in the center.

As promised in section 3.2, the static assumption on $\mathrm{H}_{2}^{+}$molecular ions is to be tested. In Fig. 10 the axial profile of the mean free path for the destruction of $\mathrm{H}_{2}^{+}$(based on 
reactions (9) and (10), Tab. 2) molecular ions is plotted for the reference case (a) for two different radii. The figure shows that this mean free path is well below $1 \mathrm{~mm}$ throughout the profile. This is smaller compared to the cell size for most of the length of the beam, except for a thin region close to the target plate, where the cell size becomes low. However, here also $T_{e}$ becomes low, and there is not much $\mathrm{H}_{2}^{+}$formed in the first place. The $T_{e}$ gradient length is in the order of $10 \mathrm{~cm}$ throughout most of the simulation domain. The picture does not change for other simulation cases or other radii. It is concluded that the quasi-static assumption is satisfied throughout the beam in the simulations presented here.

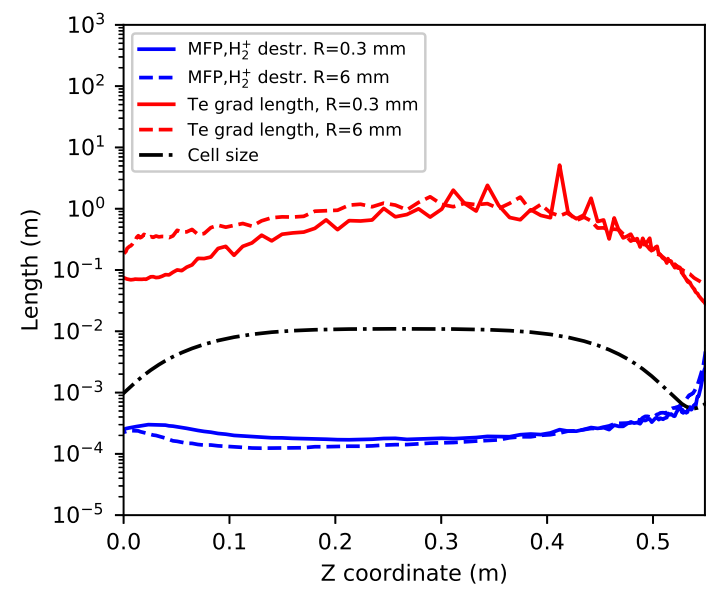

Figure 10: Parallel profiles of mean free paths for the destruction of $\mathrm{H}_{2}^{+}$molecular ions compared to the cell size at that given point, and also $T_{e}$ parallel gradient lengths for different radii.

\subsection{Comparison with experiment: Scanning neutral background pressure}

In order to see whether we can reproduce basic experimental trends, we simulated part of a background pressure scan experiment described in [39]. In this experiment, the effect of the background pressure in the vessel $P_{n}$ on the plasma beam was investigated, by changing the pumping speed. In the simulations, $P_{n}$ was changed by tweaking the albedo of the pumping surface at the back of the vessel, Fig. 2. The parameters used in the simulations are presented in Tab. 3. In the last column of the table, the background pressure is listed for each case: this is not an input parameter, but a result of the simulation. Numerical instabilities in the code related to the ion conversion reaction (7) have so far prevented us to converge cases with $P_{n}$ higher than $6.0 \mathrm{~Pa}$. To be more specific, it was found that by removing reaction (7), higher pressures can be reached. 

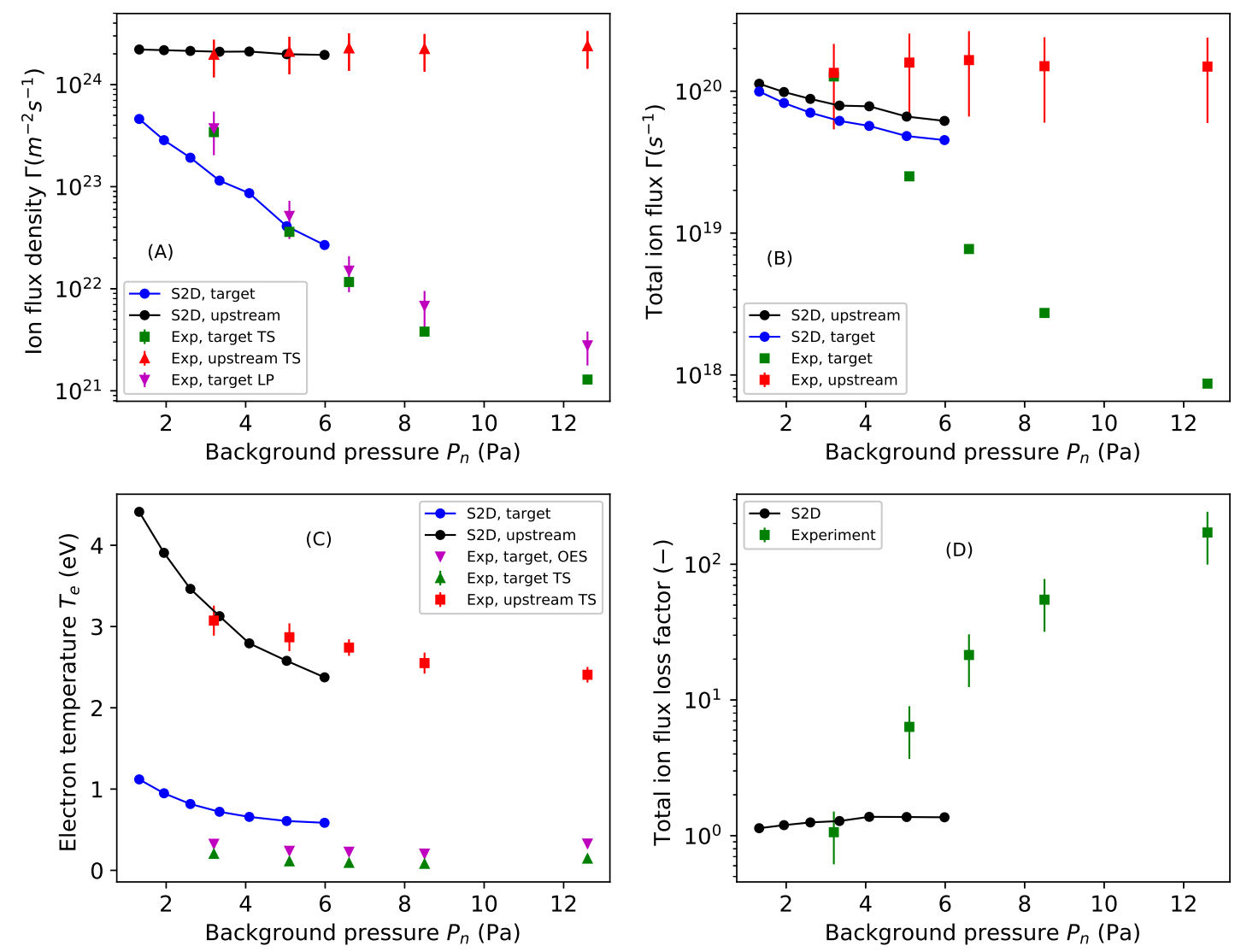

Figure 11: Comparison of the peak flux density for a given field line (A), total ion flux (B) and $T_{e}(\mathrm{C})$ between simulations and estimated from available diagnostics for the $P_{n}$ scan.

The simulation results will be analyzed in more detail in the following section. Here, we focus on comparing these simulations with the background pressure scan experiment.

The most striking finding resulting from this comparison is shown in Fig. 11 (C). The code is unable to reproduce the low temperatures found in the experiment (using spectroscopy and Thomson scattering), and this is even true for the reference case (the case where $P_{n}=3.2 \mathrm{~Pa}$ ), i.e. the one used for matching the upstream profiles. The electron temperature in the simulations seems to saturate at a level of $\sim 0.7 \mathrm{eV}$. This happens regardless of the assumed value of the radial transport coefficient $D$. We think that this affects the particle balance, leading to further discrepancies, which we address in the following.

In Fig. 11 (A), the flux density at the target is plotted, measured by a Langmuir probe embedded in the target and recalculated from the Thomson scattering (assuming that the density at the TS target position is equal to the density at the sheath edge). 
Both from the TS and LP a strong reduction of the flux density with the background pressure is found.

For completeness, the upstream flux density is also plotted, both from the code and recalculated from upstream TS. However, it is important to note that the experimental value is just a rough estimate, assuming an upstream Mach number between 0.2 and 0.7 (these were typical values measured in Pilot-PSI in [27]). These bounds give the associated error bars shown for the upstream flux in Fig. 11 (A) and (B). However, even such a large uncertainty cannot prevent from concluding that the flux density is strongly reduced at the target.

The approach from the previous section, where we compare flux densities, cannot give conclusions on the global particle balance, e.g. on the importance of recombination, as a reduction of flux density can be driven also by radial transport. Therefore, in Fig. 11 (B), the section-integrated particle flux is plotted, again as a function of $P_{n}$, from the target and upstream TS profiles and from the simulation result. For the calculation of the fluxes from the TS profiles, the same approach as in the previous section was used. Figure 11 (D) shows the ratio of the upstream total ion flux to the target total ion flux for both experiment and simulation. Experimentally, the integrated fluxes are strongly reduced for cases where $P_{n}>4.0 \mathrm{~Pa}$, even given the experimental uncertainty on the upstream flux. This indicates that there is strong volume recombination occurring between the upstream and target locations for these cases. The presence of high-n Balmer line radiation also indicates a recombining regime. High-n states are dominatly populated by electron-ion recombination [18] and are considered to be a signature of this process [40]. A typical experimental spectrum is plotted in Fig. 12 with Balmer lines up to $n=14$ clearly visible. Visually, the color of the plasma was blue in the near-target region, characteristic of a recombining region, instead of pink/red, which is typical for ionizing plasma. The spectroscopic investigation showed Balmer lines only, from which we infer that the impurity content in the plasma is low and that impurity radiation does not constitute an important energy loss channel in the studied plasmas.

The code, on the other hand, does not show a strong drop in the integrated particle flux at the target. The integrated upstream particle flux is also decreasing in the simulations. The strong local reduction found in Fig. 11 (A) and weak reduction of the total particle flux in Fig. 11 (B) indicate that the assumed radial transport coefficients chosen might be too high for the cases studied here. However, the sensitivity study performed in section 4.1 indicates that beam-width effects arising from changing the radial transport coefficient are not significant, as they tend to impact the density more than $T_{e}$, the latter being the important driver for recombination.

As rate coefficients for atomic and molecular processes, e.g. recombination, are a strong functions of $T_{e}$, we also compare the $T_{e}$ from the code and the experiment. For the target location, apart from TS, $T_{e}$ was also determined using optical emission spectroscopy, using a Boltzmann plot method on high-n Balmer lines. In the experiment, both upstream and target $T_{e}$ are weak functions of $P_{n}$. Upstream, $T_{e} \sim 2.5-3.0 \mathrm{eV}$, and at the target, 
$T_{e} \sim 0.1-0.3 \mathrm{eV}$. In the code, the upstream $T_{e}$ is more sensitive to increasing $P_{n}$, but at the target, $T_{e}$ appears to decrease only slightly, and is higher by about a factor $\sim 3$ than values measured experimentally. Typically (e.g. based on Fig. 3), one would expect the plasma to be strongly recombining also at sub $1 \mathrm{eV}$ temperatures predicted by the simulations, but Fig. 11 (B) shows that this is not the case. The reason for this can be illustrated by comparing typical recombination time scales. For the electron-ion recombination process (11), Tab. 2, a density of $5 \times 10^{19}$ and $T_{e}=0.7 \mathrm{eV}$, the recombination time $\tau_{\text {rec }}=7.8$ ms. The typical particle transit time, between upstream and target, calculated as $\tau_{\|}=$ $\int_{u}^{t} 1 / u_{\|} \mathrm{d} z$, where $u_{\|}$is the parallel fluid velocity and $z$ is the parallel coordinate. The parallel transit time for the highest $P_{n}$ case $(\mathrm{j})$ obtained in the simulations is $\sim 0.3 \mathrm{~ms}$, and is lower for the cases with lower $P_{n}$. Therefore, the ion electron pairs simply do not get enough time to recombine via channel (11) under these conditions. If $T_{e}$ were $\sim 0.2 \mathrm{eV}$, $\tau_{\text {rec }}=0.2 \mathrm{~ms}$, indicating that the recombination would become important. This shows that conclusions on the global importance of recombination cannot be based on arguments related to the local $T_{e}$ or simply on the dominance of certain rate coefficients, but rather the whole transport picture, including the macroscopic flow has to be considered.
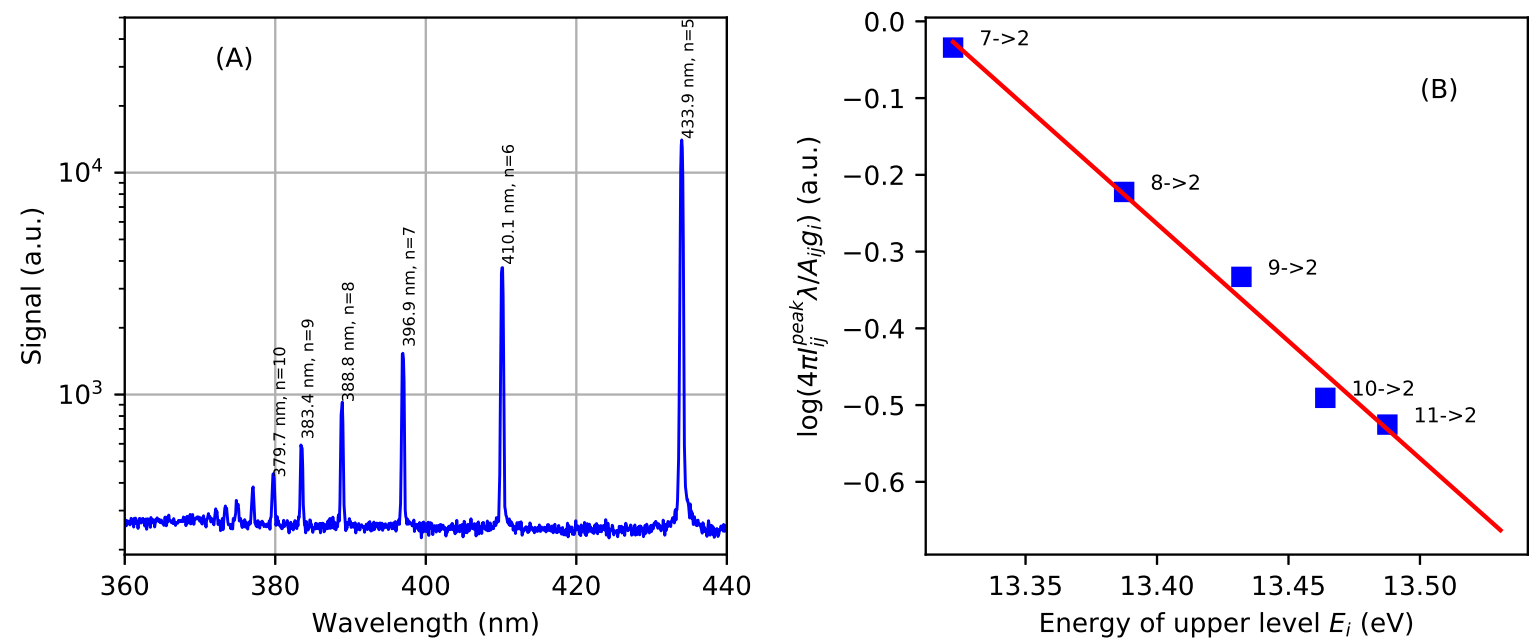

Figure 12: (A) Typical experimental spectrum of the near target plasma from a visible spectrometer looking at the near-target plasma showing high-n Balmer line radiation and (B) an example of a Boltzmann plot using lines transitions 7-2 through 11-2, yielding a temperature of $0.3 \mathrm{eV}$ in this particular case.

Concerning the $\mathrm{H}_{2}^{+}$branch of the MAR pathway, the situation is more complicated, since the recombination rate depends on the rate of formation of $\mathrm{H}_{2}^{+}$via ion conversion, which decreases as a function of $T_{e}$, and also on the ratio of the rates of the dissociative recombination (10) and of the competing, purely dissociative process (9), which is stronger 
for $T_{e}>1 \mathrm{eV}$. In any case, these processes are included in the framework of the code and they are not causing significant recombination.

\subsection{Two-point analysis of simulation results}

In order to analyse the resulting profiles more deeply, we apply a 2-point formatting analysis inspired by similar analyses performed in [41-43]. The aim here is to do the "book keeping" between different processes driving the gradients in the profiles of the particle flux density $\Gamma=n v$, total plasma pressure $\Pi=n m_{i} v_{i}^{2}+n_{i} k T_{i} / e+n_{e} k T_{e} / e$ and the parallel heat flux density for electrons $q_{e}$ and ions $q_{i}$. To identify which terms contribute the most to the reduction of e.g. the particle flux, we integrate the continuity equation 1 between an upstream position, denoted " $\mathrm{u}$ " and a position close to the target, denoted "t". Further on, these two positions correspond to upstream and target TS positions, i.e. $\mathrm{Z}=4 \mathrm{~cm}$ and $54 \mathrm{~cm}$, respectively. Next, we formally look at the radial transport term $\vec{\nabla} \cdot\left(D \vec{\nabla}_{\perp} n\right)$ on the R.H.S. of the equation as an additional source term and denote it $S_{n}^{\perp}$. Taking into account the simple, orthogonal geometry of the linear device and assuming steady state (time dependent term vanishes, which is the case in a converged simulation), we obtain the following:

$$
\Gamma_{t}-\Gamma_{u}=\int_{u}^{t} S_{n}^{(\perp)} \mathrm{d} z+\int_{u}^{t} S_{n}^{(N)} \mathrm{d} z
$$

where $\Gamma$ is the parallel particle flux density. The external source term $S_{n}^{(\text {ext })}$ from equation 1 vanishes by definition due to the choice of the " $u$ " and " $z$ " positions. The same can be done with the momentum equation 2 , in which case we multiply the equation by the ion mass $m_{i}$ so that the quantity in the divergence on the L.H.S. is the total plasma pressure $\Pi$ defined earlier in this section. Again, we formally rename the term associated with radial transport $\vec{\nabla} \cdot\left(\nu \vec{\nabla}_{\perp} n u_{\|}\right)$as $S_{G}^{(\perp)}$. After the integration we have:

$$
\Pi_{t}-\Pi_{u}=\int_{u}^{t} m_{i} S_{G}^{(\perp)} \mathrm{d} z+\int_{u}^{t} m_{i} S_{G}^{(N)} \mathrm{d} z
$$

i.e., the reduction of the total pressure between upstream and target location for a given flux tube is driven by momentum sources (sinks) due to neutrals and radial transport.

For the ion and electron energy equations 3 and 4, we move the electron pressure gradient term $u_{\|} \nabla_{\|} n k_{B} T_{e}$ to the R.H.S. of the equation. Then, we formally rename the perpendicular energy transport term (the 1st term on the R.H.S. of equations 3, 4) as $S_{E, i}^{(\perp)}$ and $S_{E, e}^{(\perp)}$, respectively. After integration, we obtain the following:

$$
q_{t, \alpha}-q_{u, \alpha}=\int_{u}^{t} S_{E, \alpha}^{(\perp)} \mathrm{d} z+\int_{u}^{t} S_{E, \alpha}^{(N)} \mathrm{d} z \pm \int_{u}^{t} Q_{\alpha}^{(c)} \mathrm{d} z \mp \int_{u}^{t} u_{\|} \frac{\partial\left(n k_{B} T_{e}\right)}{\partial z} \mathrm{~d} z
$$


Table 4: List of potential energies (in eV) appearing in the electron energy loss terms of equation 9. The number in the superscript refers to the label of the reaction in Tab. 2 to which the potential is associated

\begin{tabular}{llllll}
$\chi_{\text {ion }}^{(1)}$ & $\chi_{\text {diss }}^{(4)}$ & $\chi_{\text {diss }}^{(5)}$ & $\chi_{\text {ion }}^{(3)}$ & $\chi_{\text {diss }}^{(8)}$ & $\chi_{\text {diss }}^{(9)}$ \\
\hline 13.6 & 10.5 & 28.1 & 15.4 & 10.5 & 25.5
\end{tabular}

where $q$ is the total heat flux density and $\alpha$ is a species index, i.e. ions or electron in our case. In case of the double sign $( \pm)$ operator, the upper and lower sign corresponds to ion and electrons, respectively. The other symbols were defined in section 3.1. The obtained equation states that the reduction of the total heat flux density between upstream and target location for a given flux tube for a given species is driven by radial transport, sources/sinks due to the interaction with neutrals, sources/sinks due to temperature equilibration and a term associated with the pressure gradient, which is in fact the combined effect of the electric field force and ion-electron friction force.

For the electron energy equation, we can go one step further, and decompose the electron energy source term due to neutrals $S_{E, e}^{(N)}$ into its different contributions (remaining in the framework of the Eirene atomic \& molecular physics model used in the simulations presented here)

$$
\begin{aligned}
S_{E, e}^{(N)}= & -\chi_{\mathrm{ion}}^{(1)} n_{e} n_{\mathrm{H}}\langle\sigma v\rangle_{\mathrm{ion}}^{(1)}-\chi_{\mathrm{diss}}^{(4)} n_{e} n_{\mathrm{H}_{2}}\langle\sigma v\rangle_{\mathrm{diss}}^{(4)}-\chi_{\mathrm{diss}}^{(5)} n_{e} n_{\mathrm{H}_{2}}\langle\sigma v\rangle_{\mathrm{diss}}^{(5)} \\
& -\chi_{\mathrm{ion}}^{(3)} n_{e} n_{\mathrm{H}_{2}}\langle\sigma v\rangle_{\mathrm{ion}}^{(3)}-\chi_{\mathrm{diss}}^{(8)} n_{e} n_{\mathrm{H}_{2}^{+}}\langle\sigma v\rangle_{\mathrm{diss}}^{(8)}-\chi_{\mathrm{diss}}^{(9)} n_{e} n_{\mathrm{H}_{2}^{+}}\langle\sigma v\rangle_{\mathrm{diss}}^{(9)} \\
& -n_{e}^{2}\langle E \sigma v\rangle_{\mathrm{rec}}^{(11)}-n_{e} n_{\mathrm{H}}\left(\langle E \sigma v\rangle^{(1)}-\chi_{\mathrm{ion}}^{(1)}\langle\sigma v\rangle_{\mathrm{ion}}^{(1)}\right),
\end{aligned}
$$

where $n_{e}, n_{H}$ and $n_{H_{2}}$ are the electron, neutral atom and neutral molecule densities, $\langle\sigma v\rangle^{(\mathrm{i})}$ are rate coefficients in $\mathrm{m}^{3} \mathrm{~s}^{-1}$ and the upper index (i) denotes the process they are associated to from Tab. 2 and the values $\chi^{(\mathrm{i})}$ are potential energies associated with those reactions, e.g. for the $\mathrm{H}$ ionisation reaction (1) this energy is equal to the ionisation potential, $\chi_{\text {ion }}^{(1)}=13.6 \mathrm{eV}$. The values of the other potentials are listed in Tab. 4. The quantities of the form $\langle E \sigma v\rangle^{(\mathrm{i})}$ are total energy loss rate coefficients associated with a certain process or set of processes. These loss rates are obtained from collisional radiative modelling and are readily available in the AMJUEL database (www.eirene.de). In this case, we have the energy loss rate for electron-ion recombination (penultimate term in equation 9 ), and a term associated with line radiation by atomic hydrogen, which corresponds to the last term $-n_{e} n_{\mathrm{H}}\left(\langle E \sigma v\rangle^{(1)}-\chi_{\text {ion }}^{(1)}\langle\sigma v\rangle_{\text {ion }}^{(1)}\right)$. Since the energy weighted rate coefficient $\langle E \sigma v\rangle^{(1)}$ already includes the losses due to ionisation, we have to subtract them from total energy loss since we have already included net ionisation losses in equation 9 (1st term). Therefore, the last term in equation 9 represents the net electron energy loss due to hydrogenic radiation. 

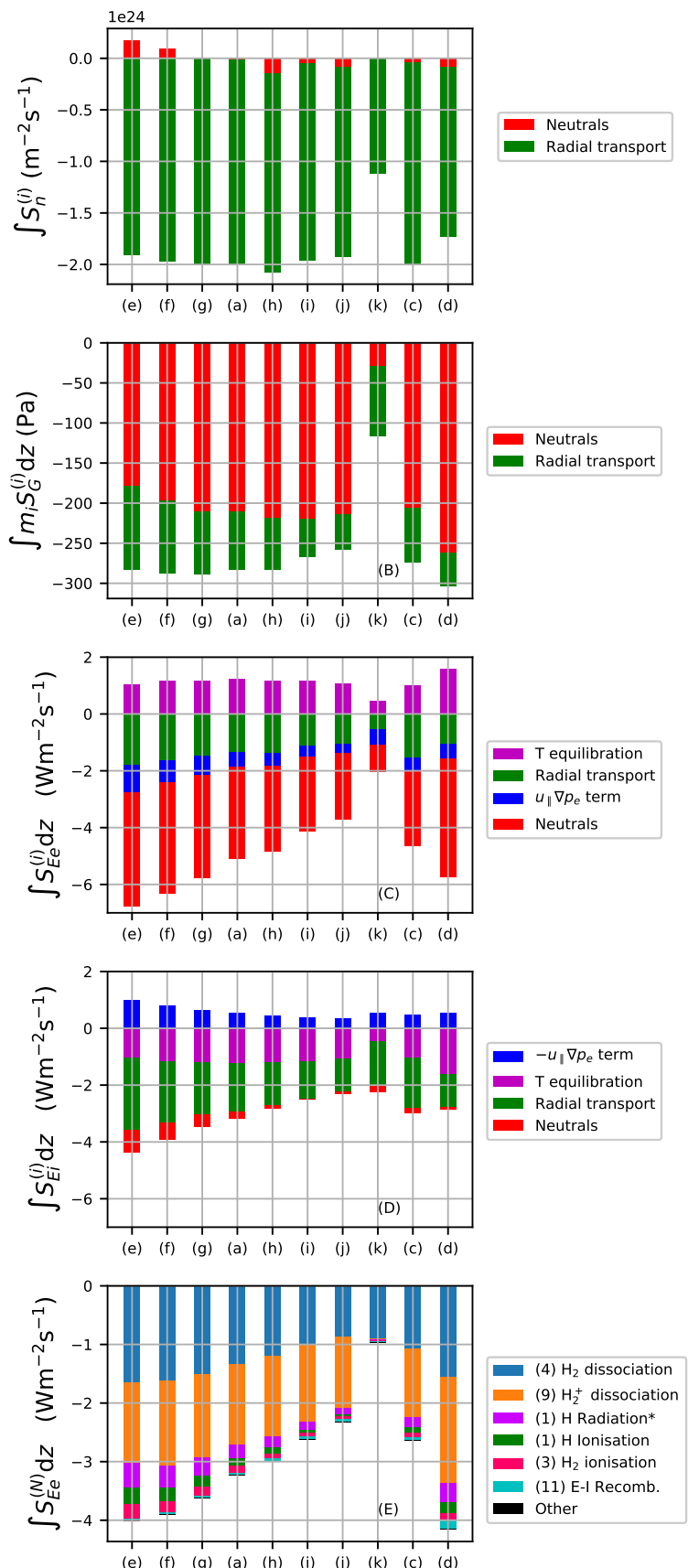

Figure 13: Bar plot of the integrated source terms for particles (A), momentum (B), electron energy (C), ion energy (D) and the energy source term due to interactions with neutrals (E) broken down into its different contributions. Detailed description of the terms are provided in the text. 
In Fig. 13 (A), the integrated loss term for particle flux density $\Gamma$ is plotted in the form of a bar chart and is split into two contributions: radial transport and sources due to neutrals, for cases (e), (f), (g), (a), (h), (i), (j), (c), (d) from Tab. 3. The cases are ordered by increasing background pressure. Case (k) uses the same setting as the reference case (a) with the exception that the elastic ion-molecule collision (process (6), Tab. 2) is switched off. The charts are for the flux tube located $1.4 \mathrm{~mm}$ off-axis. If the bar is positive, the contribution of the given process increases the flux density $\Gamma$; if it is negative, it decreases $\Gamma$. The sum of the two contributions is then equal to the difference in target and upstream flux, $\Gamma_{t}-\Gamma_{u}$. From the bar chart, it can clearly be seen that most of the reduction of the target flux density is driven by the radial transport, in every plotted case. For cases (e-g), with lower background pressure, the integrated source term due to neutrals is positive, meaning that ionisation dominates recombination. However, as $P_{n}$ is increased, it can be seen that this contribution is reduced and that the balance flips towards negative contribution for cases $(\mathrm{h}-\mathrm{j})$, meaning that in those cases recombination dominates. Still, the source terms related to neutral interactions remain comparatively small with regard to radial transport. Case $(\mathrm{k})$, where the elastic collision was switched off, is qualitatively different from the other cases. Here, the contribution of radial transport is about $\sim 2$ times smaller and the contribution of neutrals is negligible.

In Fig. 13 (B), the integrated loss term for the total pressure $\Pi$ is plotted, in a similar way as the particle flux density in the previous paragraph. In this case, the reduction of total pressure is driven mostly by interactions with neutrals, and radial transport plays a secondary role. As $P_{n}$ is increased, there is also a slight increase in the momentum sinks due to neutrals, and a reduction of the momentum sinks due to radial transport. However, in case (k), when the elastic collision (6) is switched off, the momentum sink term is significantly decreased. This indicates that elastic ion-molecule collisions are dominantly responsible for reduction of the total plasma pressure $\Pi$.

In Fig. 13 (C) and (D) the integrated loss terms for the energy flux density of electrons and ions are plotted, respectively. As pointed out earlier in this section (equation 8), the reduction in energy flux density can be driven by sources/sinks due to neutrals, radial transport, electron-ion temperature equilibration and a term related to the pressure gradient. In the case of electrons, most of the energy is dissipated by neutrals, a smaller part of it by radial transport and an even smaller fraction by the pressure gradient term. In case of the electrons, there is also a positive contribution due to the temperature equilibration term. For the reduction of ion energy flux density, the channel due to neutrals is small and energy flux dissipation due to radial transport is dominant. The temperature equilibration term has the same magnitude but opposite sign, by definition. As a general feature, the ions are transfering heat to the electrons. This is consistent with the fact that the combined energy sinks due to other processes are stronger for electrons in the regimes explored here, particularly the sinks due to neutrals. Still, the temperature equilibration term is strong enough to maintain $T_{e}=T_{i}$ throughout the axial profile, as can be seen in e.g. Fig. 7. 
It is interesting to further break down the integrated electron energy sink term due to neutrals $S_{E, e}^{(N)}$ following equation 9. This is plotted in Fig. $13(\mathrm{E})$. It can be seen that the strongest energy neutral dissipation channels are dissociation of $\mathrm{H}_{2}$ molecules (4) and $\mathrm{H}_{2}^{+}$molecular ions (9). Processes related to atomic hydrogen, like ionisation and line radiation, play a secondary role in energy dissipation. Moreover, even the atomic hydrogen results from the presence of molecules, as most of it is formed by dissociation of molecules or molecular ions.

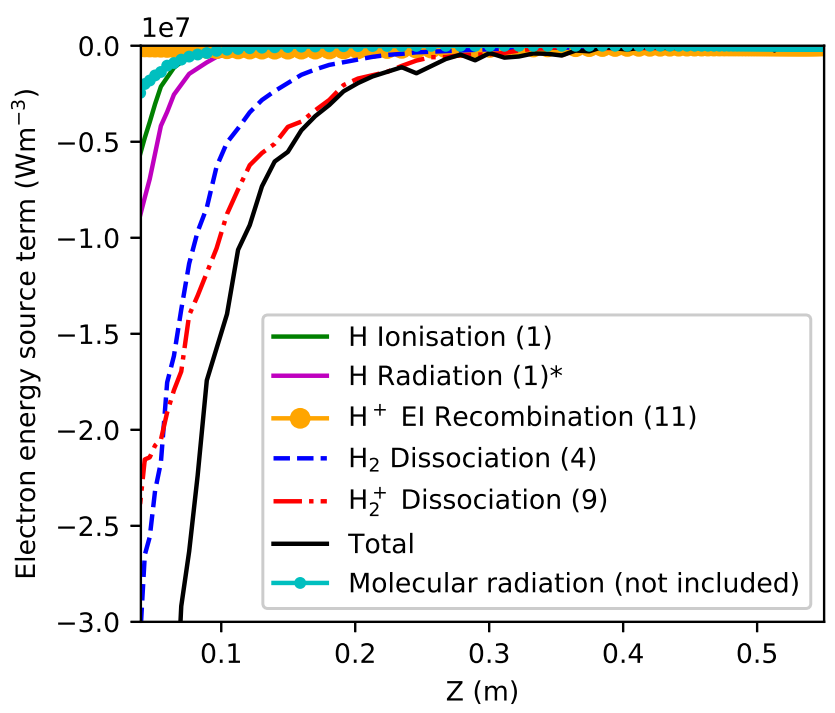

Figure 14: Parallel profiles of the total electron energy sink terms due to interactions with neutrals, following equation 9 , for the flux tube located $1.4 \mathrm{~mm}$ off axis. Only the most important contributions are plotted. Moreover, the sink term related to intrinsic molecular radiation is also plotted, in order to gauge its magnitude, but it is not included in the atomic physics framework of simulations presented here. The ${ }^{*}$ indicates that the plotted term is net radiation, with ionisation losses subtracted.

Since from the previous section we found that the simulations overestimate $T_{e}$ close to the target, we look more closely at the energy sink term to see the spatial distribution of electron energy sinks. Parallel profiles of the most important contributors to the electron energy source term due to neutrals $\left(S_{E, e}^{(N)}\right)$ are plotted for the flux tube located $1.4 \mathrm{~mm}$ from the axis of symmetry. As expected from the two-point analysis described earlier, the contributions of dissociative processes (4) and (9) are the most important energy sinks, followed by radiation and ionisation of atomic hydrogen. However, these processes seem to be efficient only in the upstream region $(Z<0.25 \mathrm{~m})$, where $T_{e}$ is sufficiently high. For $Z>0.3 \mathrm{~m}$, the electron energy sinks are negligible. We infer that the reason why the $T_{e}$ decreases rapidly in the upstream region (Fig. 8) is this strong spatial localisation of the energy sinks. Conversely, $T_{e}$ in the region closer to the target decreases only slightly. How- 
ever, $T_{e}$ measured experimentally is systematically lower than in the simulations. This indicates that there could be missing energy dissipation channels in the simulations. One possibility could be the intrinsic radiation of molecules themselves (e.g. due to molecular lines/bands) which is not included in the current framework. An energy-weighted rate coefficient is available for this kind of loss process in the AMJUEL database under reference H10.2.2.h2r, obtained by collisional-radiative modelling. This term was used to simply calculate the energy loss from the resulting $n_{e}, n_{\mathrm{H}_{2}}$ and $T_{e}$ (i.e. not in a self-consistent way) of the simulation result shown in Fig. 14. It turns out that this term is approximately two orders of magnitude lower compared to the sum of the other channels, and is also spatially localized at the upstream region. Therefore, it is considered unlikely that proper inclusion of intrinsic molecular radiation would alter the $T_{e}$ profiles significantly. Other possible dissipation mechanisms should be considered to resolve this discrepancy. It is also important to stress here that the vibrational distribution of neutral hydrogen molecules is described in a simplified way. In particular, individual vibrationally excited species are not followed, but a vibrational distribution as a function of the local $T_{e}$ is assumed based on [44], and this distribution is used when calculating rate coefficients for i.e. the ion conversion reaction (7). Therefore, the energy costs associated with vibrational excitations are also not accounted for, and it is speculated that these additional energy sinks could contribute to the reduction of $T_{e}$.

We also point out here that electric currents and drifts were not included in the simulations presented here. It is difficult to make statements about their impact without running the simulations with their inclusion. However, currents flowing through a plasma are expected to act as additional heating mechanism via Ohmic heating, especially for low temperature plasmas. Additional heating would lead to an even higher $T_{e}$ at the target, not helping resolve the observed discrepancy.

\subsection{Implications for divertors}

Implications of results from linear devices for tokamak divertors have to considered carefully due to the differences in geometry and operation. However, we think that the findings presented here can still provide some useful insights. In the previous section, it has been shown that inelastic collisions of the electrons with the surrounding molecules provide a strong heat dissipation channel, accounting for $>50 \%$ of the reduction of the total heat flux density (Fig. 13) for a given flux tube. It is important to point out that in Pilot-PSI a large part of the molecules originate from the cascaded arc source, since only a fraction (typically $\sim 10 \%$ ) is ionised. In tokamaks this is not the case, since the neutrals in the divertor are exclusively supplied by the plasma recycling itself, i.e. via target plate neutralisation and volume recombination of ions and electrons and further recombination of the resulting atoms into molecules. In general, the molecule density is typically lower than the atomic density and it is concentrated close to the target plates, especially for open divertor configurations. However, recent modelling efforts of closed divertor geometries 
at DIII-D [45-47] have shown molecular densities comparable to Pilot-PSI conditions, up to $10^{20} \mathrm{~m}^{-3}$ and a near-target $T_{e} \sim 1 \mathrm{eV}$. Such refinements of the divertor geometry have resulted in better performance in terms of power dissipation, both from the edge transport codes and modelling and experiments. Similar results have been obtained by modelling of the super-X divertor for MAST-upgrade [48], which has a very closed divertor wall geometry and is expected to operate at high molecular pressures. It is expected that in such divertor conditions, the principle acting mechanisms in terms of atomic and molecular physics, will be the same. In our setup of the Soledge2D-Eirene transport code, comparisons with modelling and experiments show that $T_{e}$ close to the target is overestimated by the code under the examined, low $T_{e}$, high $n_{e}$ conditions.

\section{Conclusion \& Outlook}

The Soledge2D-Eirene tokamak edge plasma transport code has been applied in the cylindrical geometry of the Pilot-PSI linear plasma device with the aim to a) assess how well the code can reproduce experimental trends and b) give new insights into the interpretation of experiments. The effect of the neutral pressure $P_{n}$ on the simulation results was investigated and compared to measurements using TS, an embedded LP in the target and visible spectroscopy. It has been found that in the simulations, $T_{e}$ at the target is overestimated with respect to the measurements using TS and spectroscopy. $T_{e}$ in the simulations appears to saturate at $0.7 \mathrm{eV}$ for a wide range of parameters (also in response to changes of the perpendicular transport coefficients, the main unknown parameter in the simulations), while experimentally values of $0.1-0.3 \mathrm{eV}$ are found. It is inferred that the overestimation of $T_{e}$ in the simulations is the cause for the underestimation of volume recombination, which is a strong function of $T_{e}$ for $T_{e}<1 \mathrm{eV}$.

A two-point formatting approach was used to analyse the drivers of the reduction of flux density, total plasma pressure and the heat flux density in the simulations between upstream and target locations. It was found that the strongest driver for reduction of the particle flux density on a given flux tube was radial transport. For the reduction of total plasma pressure, elastic ion-molecule collisions are the dominant mechanism. For the heat, inelastic collisions between electrons and neutral backround particles dissipate most of the heat flux. From these inelastic processes, dissociation of molecules and molecular ions were found to be the strongest contributors. However, these energy sinks are located in the upstream region, where $T_{e}$ is high enough to efficiently break up molecules and molecular ions; in locations close to the target, the simulations do not predict any mechanism that could dissipate the heat flux and subsequently reduce $T_{e}$ to values of $0.2 \mathrm{eV}$ found in the experiment, i.e. where volume recombination could remove a significant number of plasma particles. A rudimentary estimate was made to try to account for intrinsic (line/band) radiation of molecules themselves, which was not included in the neutral physics model in Eirene in the simulations presented here, however it turned out that it is unlikely 
that this process could explain the discrepancy in $T_{e}$. Other mechanism will be looked into in the future, for instance energy costs due to vibrational excitations of molecules. Moreover, the convergence issues for $P_{n}>6 \mathrm{~Pa}$ when ion conversion is present merit further investigation.

Further possibilities to study plasmas relevant to detachment are offered by the MagnumPSI linear plasma device, with a better diagnostic coverage. Target calorimetry and a newly installed bolometric diagnostic [49] could shed more light on studies of the power balance, while a collective Thomson scattering diagnostic [50] (under development) could give axial velocity information.

\section{Acknowledgments}

This work was granted access to the HPC resources of Aix-Marseille University financed by the project Equip@Meso (ANR-10-EQPX-29-01) of the program "Investissements d'Avenir" supervised by the Agence Nationale pour la Recherche. This work was carried out with financial support from NWO and was carried out within the framework of the Erasmus Mundus International Doctoral College in Fusion Science and Engineering (FUSION-DC). This work has been carried out within the framework of the EUROfusion Consortium and has received funding from the Euratom research and training programme 2014-2018 under grant agreement No 633053. The views and opinions expressed herein do not necessarily reflect those of the European Commision.

\section{References}

[1] H. Bufferand, G. Ciraolo, Y. Marandet, J. Bucalossi, P. Ghendrih, J. Gunn, N. Mellet, P. Tamain, R. Leybros, N. Fedorczak, F. Schwander, and E. Serre, "Numerical modelling for divertor design of the WEST device with a focus on plasma-wall interactions," Nuclear Fusion, vol. 55, no. 5, 2015.

[2] D. Reiter, M. Baelmans, and P. Börner, "The eirene and B2-eirene codes," Fusion Science and Technology, vol. 47, no. 2, pp. 172-186, 2005.

[3] T. Eich, B. Sieglin, A. Scarabosio, W. Fundamenski, R. J. Goldston, and A. Herrmann, "Inter-ELM power decay length for JET and ASDEX Upgrade: Measurement and comparison with heuristic drift-based model," Physical Review Letters, vol. 107, no. $21,2011$.

[4] R. A. Pitts, A. Kukushkin, A. Loarte, A. Martin, M. Merola, C. E. Kessel, V. Komarov, and M. Shimada, "Status and physics basis of the ITER divertor," in Physica Scripta T, vol. T138, 2009. 
[5] S. I. Krasheninnikov, A. S. Kukushkin, and A. A. Pshenov, "Divertor plasma detachment," Physics of Plasmas, vol. 23, no. 5, 2016.

[6] S. I. Krasheninnikov and A. S. Kukushkin, "Physics of ultimate detachment of a tokamak divertor plasma," Journal of Plasma Physics, vol. 83, no. 05, p. 155830501 , 2017.

[7] G. F. Matthews, "Plasma detachment from divertor targets and limiters," Journal of Nuclear Materials, vol. 220-222, pp. 104-116, apr 1995.

[8] W. L. Hsu, M. Yamada, and F. H. Tenney, "Neutral gas blanket effects in a gaseous divertor," Journal of Nuclear Materials, vol. 111-112, no. C, pp. 311-316, 1982.

[9] W. L. Hsu, M. Yamada, and P. J. Barrett, "Experimental simulation of the gaseous tokamak divertor," Physical Review Letters, vol. 49, no. 14, pp. 1001-1004, 1982.

[10] L. Schmitz, R. Lehmer, G. Chevalier, G. Tynan, P. Chia, R. Doerner, and R. W. Conn, "Experimental simulation of the gaseous divertor concept in PISCES-A," Journal of Nuclear Materials, vol. 176-177, no. C, pp. 522-527, 1990.

[11] N. Ohno, N. Ezumi, S. Takamura, S. I. Krasheninnikov, and A. Y. Pigarov, "Experimental evidence of molecular activated recombination in detached recombining plasmas," Physical Review Letters, vol. 81, no. 4, pp. 818-821, 1998.

[12] N. Ezumi, D. Nishijima, H. Kojima, N. Ohno, S. Takamura, S. I. Krasheninnikov, and A. Y. Pigarov, "Contribution of molecular activated recombination to hydrogen plasma detachment in the divertor plasma simulator NAGDIS-II," Journal of Nuclear Materials, vol. 266, pp. 337-342, 1999.

[13] D. Nishijima, U. Wenzel, K. Ohsumi, N. Ohno, Y. Uesugi, and S. Takamura, "Characteristics of detached plasmas associated with electron-ion and molecular assisted recombinations in NAGDIS-II," Plasma Physics and Controlled Fusion, vol. 44, no. 5, pp. 597-610, 2002.

[14] A. Y. Pigarov and S. I. Krasheninnikov, "Application of the collisional-radiative, atomic-molecular model to the recombining divertor plasma," Physics Letters, Section A: General, Atomic and Solid State Physics, vol. 222, no. 4, pp. 251-257, 1996.

[15] E. M. Hollmann, D. G. Whyte, D. Nishijima, N. Ohno, Y. Uesugi, and N. Ezumi, "Evidence for the importance of radial transport in plasma detachment in the Nagoya University Divertor Simulator (NAGDIS-II)," Physics of Plasmas, vol. 8, no. 7, pp. 3314-3320, 2001. 
[16] N. Ohno, D. Nishijima, S. Takamura, Y. Uesugi, M. Motoyama, N. Hattori, H. Arakawa, N. Ezumi, S. Krasheninnikov, A. Pigarov, and U. Wenzel, "Static and dynamic behaviour of plasma detachment in the divertor simulator experiment NAGDIS-II," Nuclear Fusion, vol. 41, no. 8, pp. 1055-1065, 2001.

[17] N. Ohno, "Plasma detachment in linear devices," Plasma Physics and Controlled Fusion, vol. 59, no. 3, 2017.

[18] D. Lumma, J. L. Terry, and B. Lipschultz, "Radiative and three-body recombination in the Alcator C-Mod divertor," Physics of Plasmas, vol. 4, no. 7, pp. 2555-2566, 1997.

[19] J. L. Terry, B. Lipschultz, A. Y. Pigarov, S. I. Krasheninnikov, B. LaBombard, D. Lumma, H. Ohkawa, D. Pappas, and M. Umansky, "Volume recombination and opacity in Alcator C-Mod divertor plasmas," Physics of Plasmas, vol. 5, no. 5, pp. 1759-1766, 1998.

[20] R. C. Wieggers, D. P. Coster, P. W. C. Groen, H. J. De Blank, and W. J. Goedheer, "B2.5-Eunomia simulations of Pilot-PSI plasmas," Journal of Nuclear Materials, vol. 438, no. SUPPL, 2013.

[21] M. Baeva, W. J. Goedheer, N. J. Lopes Cardozo, and D. Reiter, "B2-EIRENE simulation of plasma and neutrals in MAGNUM-PSI," Journal of Nuclear Materials, vol. 363-365, no. 1-3, pp. 330-334, 2007.

[22] H. J. Van Eck, T. Abrams, M. A. Van Den Berg, S. Brons, G. G. Van Eden, M. A. Jaworski, R. Kaita, H. J. Van Der Meiden, T. W. Morgan, M. J. Van De Pol, J. Scholten, P. H. Smeets, G. De Temmerman, P. C. De Vries, and P. A. Zeijlmans Van Emmichoven, "Operational characteristics of the high flux plasma generator Magnum-PSI," Fusion Engineering and Design, vol. 89, no. 9-10, pp. 2150-2154, 2014.

[23] H. Kastelewicz and G. Fussmann, "Plasma modelling for the PSI linear plasma device," Contributions to Plasma Physics, vol. 44, no. 4, pp. 352-360, 2004.

[24] M. S. Islam, Y. Nakashima, and A. Hatayama, "Investigation of plasma behavior during noble gas injection in the end-cell of GAMMA 10/PDX by using the multifluid code 'LINDA'," Plasma Physics and Controlled Fusion, vol. 59, no. 12, 2017.

[25] B. de Groot, R. S. Al, R. Engeln, W. J. Goedheer, O. G. Kruijt, H. J. Meiden, P. R. Prins, D. C. Schram, P. H. Smeets, V. P. Veremiyenko, W. A. Vijvers, J. Westerhout, A. W. Kleyn, N. J. Cardozo, and G. J. van Rooij, "Extreme hydrogen plasma fluxes at Pilot-PSI enter the ITER divertor regime," Fusion Engineering and Design, vol. 82, no. 15-24, pp. 1861-1865, 2007. 
[26] W. A. Vijvers, C. A. Van Gils, W. J. Goedheer, H. J. Van Der Meiden, D. C. Schram, V. P. Veremiyenko, J. Westerhout, N. J. Lopes Cardozo, and G. J. Van Rooij, "Optimization of the output and efficiency of a high power cascaded arc hydrogen plasma source," Physics of Plasmas, vol. 15, no. 9, 2008.

[27] G. J. Van Rooij, V. P. Veremiyenko, W. J. Goedheer, B. De Groot, A. W. Kleyn, P. H. Smeets, T. W. Versloot, D. G. Whyte, R. Engeln, D. C. Schram, and N. J. Cardozo, "Extreme hydrogen plasma densities achieved in a linear plasma generator," Applied Physics Letters, vol. 90, no. 12, 2007.

[28] H. J. Van Der Meiden, A. R. Lof, M. A. Van Den Berg, S. Brons, A. J. Donné, H. J. Van Eck, P. M. Koelman, W. R. Koppers, O. G. Kruijt, N. N. Naumenko, T. Oyevaar, P. R. Prins, J. Rapp, J. Scholten, D. C. Schram, P. H. Smeets, G. Van Der Star, S. N. Tugarinov, and P. A. Van Emmichoven, "Advanced Thomson scattering system for high-flux linear plasma generator," Review of Scientific Instruments, vol. 83, no. 12, 2012.

[29] J. P. Gunn, "The influence of magnetization strength on the sheath: Implications for flush-mounted probes," Physics of Plasmas, vol. 4, no. 12, pp. 4435-4446, 1997.

[30] H. Bufferand, C. Baudoin, J. Bucalossi, G. Ciraolo, J. Denis, N. Fedorczak, D. Galassi, P. Ghendrih, R. Leybros, Y. Marandet, N. Mellet, J. Morales, N. Nace, E. Serre, P. Tamain, and M. Valentinuzzi, "Implementation of drift velocities and currents in SOLEDGE2DEIRENE," Nuclear Materials and Energy, vol. 12, pp. 852857, 2017.

[31] H. Bufferand, B. Bensiali, J. Bucalossi, G. Ciraolo, P. Genesio, P. Ghendrih, Y. Marandet, A. Paredes, F. Schwander, E. Serre, and P. Tamain, "Near wall plasma simulation using penalization technique with the transport code SOLEDGE2DEIRENE," Journal of Nuclear Materials, vol. 438, no. SUPPL, 2013.

[32] L. Isoardi, G. Chiavassa, G. Ciraolo, P. Haldenwang, E. Serre, P. Ghendrih, Y. Sarazin, F. Schwander, and P. Tamain, "Penalization modeling of a limiter in the Tokamak edge plasma," Journal of Computational Physics, vol. 229, no. 6, pp. 2220$2235,2010$.

[33] A. Paredes, H. Bufferand, G. Ciraolo, F. Schwander, E. Serre, P. Ghendrih, and P. Tamain, "A penalization technique to model plasma facing components in a tokamak with temperature variations," Journal of Computational Physics, vol. 274, pp. 283-298, 2014.

[34] B. Bensiali, G. Chiavassa, and J. Liandrat, "Penalization of Robin boundary conditions," Applied Numerical Mathematics, vol. 96, pp. 134-152, 2015. 
[35] V. Kotov, D. Reiter, R. A. Pitts, S. Jachmich, A. Huber, and D. P. Coster, "Numerical modelling of high density JET divertor plasma with the SOLPS4.2 (B2-EIRENE) code," Plasma Physics and Controlled Fusion, vol. 50, no. 10, 2008.

[36] A. S. Kukushkin, H. D. Pacher, V. Kotov, D. Reiter, D. Coster, and G. W. Pacher, "Effect of neutral transport on ITER divertor performance," Nuclear Fusion, vol. 45, no. 7, pp. 608-616, 2005.

[37] K. Jesko, Y. Marandet, H. Bufferand, J. Gunn, H. van der Meiden, and G. Ciraolo, "Soledge2D-Eirene simulations of the Pilot-PSI linear plasma device compared to experimental data," Contributions to Plasma Physics, vol. 58, pp. 798-804, jul 2018.

[38] N. Den Harder, D. C. Schram, W. J. Goedheer, H. J. De Blank, M. C. Van De Sanden, and G. J. Van Rooij, "Residual gas entering high density hydrogen plasma: Rarefaction due to rapid heating," Plasma Sources Science and Technology, vol. 24, no. $2,2015$.

[39] K. Ješko, H. J. van der Meiden, J. P. Gunn, J. W. Vernimmen, and G. De Temmerman, "Plasma pressure and particle loss studies in the Pilot-PSI high flux linear plasma generator," Nuclear Materials and Energy, vol. 12, pp. 1088-1093, aug 2017.

[40] R. C. Isler, G. R. McKee, N. H. Brooks, W. P. West, M. E. Fenstermacher, and R. D. Wood, "Signatures of deuterium recombination in the DIII-D divertor," Physics of Plasmas, vol. 4, no. 8, pp. 2989-2996, 1997.

[41] C. Sang, H. Y. Guo, P. C. Stangeby, L. L. Lao, and T. S. Taylor, "SOLPS analysis of neutral baffling for the design of a new diverter in DIII-D," Nuclear Fusion, vol. 57, no. $5,2017$.

[42] V. Kotov and D. Reiter, "Two-point analysis of the numerical modelling of detached divertor plasmas," Plasma Physics and Controlled Fusion, vol. 51, no. 11, 2009.

[43] P. C. Stangeby and C. Sang, "Strong correlation between D 2 density and electron temperature at the target of divertors found in SOLPS analysis," Nuclear Fusion, vol. 57 , no. 5, 2017.

[44] T. Greenland and D. Reiter, "The Role of Molecular Hydrogen in Plasma Recombination," Report, vol. JUEL-3258, no. FZ-Juelich, 1996.

[45] C. F. Sang, P. C. Stangeby, H. Y. Guo, A. W. Leonard, B. Covele, L. L. Lao, A. L. Moser, and D. M. Thomas, "SOLPS modeling of the effect on plasma detachment of closing the lower divertor in DIII-D," Plasma Physics and Controlled Fusion, vol. 59, no. $2,2017$. 
[46] C. Sang, H. Y. Guo, P. C. Stangeby, L. L. Lao, and T. S. Taylor, "SOLPS analysis of neutral baffling for the design of a new diverter in DIII-D," Nuclear Fusion, vol. 57, no. 5, 2017.

[47] L. Casali, C. Sang, A. Moser, B. Covele, H. Guo, and C. Samuell, "Modelling the effect of divertor closure on detachment onset in DIII-D with the SOLPS code," Contributions to Plasma Physics, vol. 58, pp. 725-731, jul 2018.

[48] E. Havl????kov??, M. Wischmeier, and G. Fishpool, "Modelling the Effect of the Super-X Divertor in MAST Upgrade on Transition to Detachment and Distribution of Volumetric Power Losses," Contributions to Plasma Physics, vol. 54, no. 4-6, pp. 448-453, 2014.

[49] G. van Eden, M. Reinke, S. Brons, G. van der Bijl, B. Krijger, R. Lavrijsen, S. Huber, R. Perillo, M. van de Sanden, and T. Morgan, "Plasma radiation studies in MagnumPSI using resistive bolometry," Nuclear Fusion, vol. 58, p. 106006, oct 2018.

[50] H. J. Van der Meiden, J. W. Vernimmen, K. Bystrov, K. Jesko, M. Y. Kantor, G. De Temmerman, and T. W. Morgan, "Collective Thomson scattering system for determination of ion properties in a high flux plasma beam," Applied Physics Letters, vol. 109, no. 26, 2016. 